\title{
Probing quadruplet scalar dark matter at current and future $p p$ colliders
}

\author{
Yu-Pan Zeng $\odot$, Chengfeng Cai, Dan-Yang Liu, Zhao-Huan Yu๑, ${ }^{*}$ and Hong-Hao Zhang $\oplus^{\dagger}$ \\ School of Physics, Sun Yat-Sen University, Guangzhou 510275, China
}

(Received 28 October 2019; accepted 11 June 2020; published 24 June 2020)

\begin{abstract}
We investigate a dark matter model involving an inert $\mathrm{SU}(2)_{\mathrm{L}}$ quadruplet scalar with hypercharge $1 / 2$. After the electroweak symmetry breaking, the dark sector contains one doubly charged, two singly charged, and two neutral scalars. The lighter neutral scalar can be a viable dark matter candidate. Electroweak production of these scalars at the Large Hadron Collider leads to potential signals in the monojet $+\mathbb{E}_{\mathrm{T}}$ and soft - leptons + jets $+E_{\mathrm{T}}$ channels. We thus derive constraints on the model by reinterpreting recent experimental searches. Based on simulation, we further evaluate the sensitivity at a future $100 \mathrm{TeV} p p$ collider.
\end{abstract}

DOI: $10.1103 /$ PhysRevD.101.115033

\section{INTRODUCTION}

Among various candidates of particle dark matter (DM), weakly interacting massive particles (WIMPs) seem rather appealing, because they could naturally predict a thermal relic abundance consistent with the observed value [1-3]. It is straightforward to construct WIMP models by extending the standard model $(\mathrm{SM})$ with new colorless $\mathrm{SU}(2)_{\mathrm{L}}$ multiplets in the dark sector [4-33], which have electroweak interaction strength by definition. The DM candidate in such models arises from the electrically neutral components of the multiplets.

If the DM candidate is a scalar particle, the minimal extension is to introduce an inert $\mathrm{SU}(2)_{\mathrm{L}}$ doublet scalar with hypercharge $Y=1 / 2$, resulting in the inert doublet model (IDM) [34-37]. The term "inert" means that there exists an unbroken $Z_{2}$ symmetry that forbids the doublet gaining a nonzero vacuum expectation value (VEV) and directly coupling to SM fermions. Consequently, if the lightest component of the doublet is one of the electrically neutral components, it would be stable, acting as a WIMP DM candidate. A next-to-minimal model can be constructed with an inert triplet scalar of $Y=0$ or $Y=1$ [38-42].

In this paper, we go further to study a scalar DM model with an inert quadruplet scalar of $Y=1 / 2[10,19]$, dubbed the quadruplet scalar dark matter (QSDM) model, which has been much less investigated in the past. The study in

\footnotetext{
*yuzhaoh5@mail.sysu.edu.cn

zhh98@mail.sysu.edu.cn
}

Published by the American Physical Society under the terms of the Creative Commons Attribution 4.0 International license. Further distribution of this work must maintain attribution to the author(s) and the published article's title, journal citation, and DOI. Funded by SCOAP.
Ref. [10] focused on how this model can support a strong first-order electroweak phase transition, as well as the constraints from electroweak oblique parameters, invisible Higgs decay, direct DM detection, and relic abundance. In our previous work [19], we investigated the projected sensitivity to this model from improved determination of electroweak oblique parameters in the future Circular Electron-Positron Collider (CEPC) project [43]. In this work, we concentrate on production signals of the new scalar bosons in the model at the Large Hadron Collider (LHC) and future $p p$ colliders, which have not been studied in the previous literature.

In the QSDM model, there are three types of independent quartic couplings between the quadruplet and the SM Higgs doublet, which contribute to the mass terms of the quadruplet components due to the nonzero Higgs VEV. As a result, the components of the quadruplet are split in mass. Mass eigenstates in the dark sector include two neutral scalars, two singly charged scalars, and one doubly charged scalar. The lighter neutral scalar could be a viable DM candidate. DM scattering off nuclei can be mediated by the Higgs boson through the quartic couplings, leading to possible signals in direct detection experiments.

Moreover, the dark sector scalars could be produced in pairs at the LHC via electroweak gauge interactions. Because of the $Z_{2}$ symmetry, all these scalars finally decay into the DM particle, which can escape from the LHC detectors, resulting in a large missing transverse energy $\left(\mathbb{E}_{\mathrm{T}}\right)$ in the final state. Since the mass spectrum in the dark sector is typically compressed, visible decay products from the scalars tend to be soft. Therefore, a hard jet from initial state radiation may be required for triggering the signal at the LHC. Thus, one possible searching channel is the monojet $+\mathbb{E}_{\mathrm{T}}$ channel, which has been widely applied for searching dark matter [44-49]. Furthermore, additional soft leptons may contain imprints of the scalar decays [50-54]. 
This motivates us to study a soft - leptons + jets $+\boldsymbol{E}_{\mathrm{T}}$ channel well. We will estimate the related constraints on the QSDM model by reinterpreting the existed LHC searches.

At the LHC energies, electroweak production rates for the dark sector scalars are quite low, and, hence, the constraints from current LHC searches are still weak. Nevertheless, future $p p$ colliders with much higher energies have been proposed, including the Super ProtonProton Collider (SPPC) at $\sqrt{s} \sim 70-100 \mathrm{TeV}$ [55] and the $p p$ Future Circular Collider (FCC-hh) at $\sqrt{s} \sim$ $100 \mathrm{TeV}$ [56]. The increase of the collision energy makes it possible to probe much heavier electroweak scalars. We thus explore the sensitivity to the QSDM model at a $100 \mathrm{TeV} p p$ collider based on Monte Carlo simulation.

This paper is organized as follows. In Sec. II, we introduce the model details. In Sec. III, we identify the parameter regions that are consistent with the observed relic abundance and study the constraints from direct detection experiments. In Sec. IV, we explore the constraint from the LHC search in the monojet $+\boldsymbol{E}_{\mathrm{T}}$ channel, as well as the sensitivity at a $100 \mathrm{TeV} p p$ collider. In Sec. V, the soft leptons + jets $+\mathbb{E}_{\mathrm{T}}$ channel is studied. Section VI gives the conclusions and discussions.

\section{QUADRUPLET SCALAR DARK MATTER MODEL}

In the QSDM model, we introduce a $\mathrm{SU}(2)_{\mathrm{L}}$ quadruplet scalar $X$ with hypercharge $Y=1 / 2[10,19]$. We assume that $X$ is inert; i.e., $X$ is odd under a $Z_{2}$ symmetry, but all SM fields are $Z_{2}$ even. On the one hand, we can express the quadruplet in the vector notation $X=\left(X^{++}, X^{+}, X^{0}, X^{-}\right)^{\mathrm{T}}$ with explicitly indicated electric charges. On the other hand, it can be denoted by a totally symmetric $\mathrm{SU}(2)_{\mathrm{L}}$ tensor $X^{i j k}(i, j, k=1,2)$. The components in the two notations are related by

$$
X=\left(\begin{array}{c}
X^{++} \\
X^{+} \\
X^{0} \\
X^{-}
\end{array}\right)=\left(\begin{array}{c}
X^{111} \\
\sqrt{3} X^{112} \\
\sqrt{3} X^{122} \\
X^{222}
\end{array}\right) .
$$

Note that $X^{+} \neq\left(X^{-}\right)^{*}$. The neutral component $X^{0}$ can be separated into two real scalars $\phi$ and $a$ :

$$
X^{0}=\frac{1}{\sqrt{2}}(\phi+i a) .
$$

The Lagrangian in the QSDM model is given by

$$
\mathcal{L}=\mathcal{L}_{\mathrm{SM}}+\left(D_{\mu} X\right)^{\dagger} D^{\mu} X-V(X),
$$

where $\mathcal{L}_{\mathrm{SM}}$ is the SM Lagrangian and $V(X)$ is the potential involving $X$. The covariant derivative for $X$ is $D_{\mu}=$ $\partial_{\mu}-i g W_{\mu}^{a} T^{a}-i g^{\prime} B_{\mu} / 2$, where $T^{a}$ are the $\mathrm{SU}(2)_{\mathrm{L}}$ generators in the representation 4. Electroweak gauge interaction terms for the quadruplet are explicitly given in the Appendix.

Respecting the $Z_{2}$ symmetry $X^{i j k} \rightarrow-X^{i j k}$, we write down the potential $V(X)$ as

$$
\begin{aligned}
V(X)= & M_{X}^{2}|X|^{2}+\lambda_{0}|X|^{2}|H|^{2}+\lambda_{1} X_{i j k}^{\dagger} X^{i j l} H_{l}^{\dagger} H^{k} \\
& +\left(\lambda_{2} X^{i k l} X^{j m n} H_{i}^{\dagger} H_{j}^{\dagger} \epsilon_{k m} \epsilon_{l n}+\text { H.c. }\right) \\
& + \text { self-interaction terms of } X,
\end{aligned}
$$

where $H$ is the SM Higgs doublet. Here we adopt a convention $\epsilon^{12}=1=-\epsilon_{12}$ for the asymmetric tensors $\epsilon^{i j}$ and $\epsilon_{i j}$. We do not give the explicit forms for the quadruplet self-interaction terms, because they will not affect the following discussions. Note that one may write down an extra operator $X_{i j k}^{\dagger} X^{i j l} H_{m}^{\dagger} H^{n} \varepsilon^{k m} \varepsilon_{l n}$, but it is not independent, because $X_{i j k}^{\dagger} X^{i j l} H_{m}^{\dagger} H^{n} \varepsilon^{k m} \varepsilon_{l n}=$ $X_{i j k}^{\dagger} X^{i j l} H_{l}^{\dagger} H^{k}-|X|^{2}|H|^{2}$. If $\lambda_{2}$ is complex, we can always make it real by a phase redefinition of the quadruplet. Hereafter, we just use a real $\lambda_{2}$. Since the one-loop contributions to the beta function of the quartic Higgs coupling $\lambda$ from $\lambda_{0}, \lambda_{1}$, and $\lambda_{2}$ are all positive [57], the Higgs vacuum stability problem in the SM [58] would be partially alleviated in the QSDM model.

After $H$ gets its VEV $v=246.22 \mathrm{GeV}$, mass terms for the quadruplet components can be expressed as

$$
\begin{aligned}
\mathcal{L}_{\text {mass }}= & -\frac{1}{2} m_{\phi}^{2} \phi^{2}-\frac{1}{2} m_{a}^{2} a^{2} \\
& -\left(\begin{array}{ll}
\left(X^{+}\right)^{*} & X^{-}
\end{array}\right) M_{\mathrm{C}}^{2}\left(\begin{array}{c}
X^{+} \\
\left(X^{-}\right)^{*}
\end{array}\right)-m_{++}^{2}\left|X^{++}\right|^{2},
\end{aligned}
$$

with

$$
\begin{gathered}
m_{\phi}^{2}=M_{X}^{2}+\frac{1}{6}\left(3 \lambda_{0}+2 \lambda_{1}-4 \lambda_{2}\right) v^{2} \\
m_{a}^{2}=M_{X}^{2}+\frac{1}{6}\left(3 \lambda_{0}+2 \lambda_{1}+4 \lambda_{2}\right) v^{2} \\
M_{\mathrm{C}}^{2}=\left(\begin{array}{cc}
M_{X}^{2}+\left(3 \lambda_{0}+\lambda_{1}\right) v^{2} / 6 & \lambda_{2} v^{2} / \sqrt{3} \\
\lambda_{2} v^{2} / \sqrt{3} & M_{X}^{2}+\left(\lambda_{0}+\lambda_{1}\right) v^{2} / 2
\end{array}\right),
\end{gathered}
$$

$$
m_{++}^{2}=M_{X}^{2}+\frac{1}{2} \lambda_{0} v^{2} .
$$

The mass-squared matrix $M_{\mathrm{C}}^{2}$ for the singly charged scalars can be diagonalized by a $2 \times 2$ rotation matrix $O$, which satisfies 


$$
\begin{aligned}
& O^{\mathrm{T}} M_{\mathrm{C}}^{2} O=\left(\begin{array}{ll}
m_{1}^{2} & \\
& m_{2}^{2}
\end{array}\right), \\
& O=\left(\begin{array}{cc}
\cos \theta & -\sin \theta \\
\sin \theta & \cos \theta
\end{array}\right) .
\end{aligned}
$$

The rotation angel $\theta$ can be obtained from

$$
\sin \theta=\frac{-\sqrt{6} \lambda_{2}}{\sqrt{\lambda_{1}^{2}+12 \lambda_{2}^{2}+\lambda_{1} \sqrt{\lambda_{1}^{2}+12 \lambda_{2}^{2}}}} .
$$

Thus, the singly charged mass eigenstates $X_{1}^{+}$and $X_{2}^{+}$are related to the gauge eigenstates $X^{+}$and $\left(X^{-}\right)^{*}$ through

$$
\left(\begin{array}{c}
X^{+} \\
\left(X^{-}\right)^{*}
\end{array}\right)=O\left(\begin{array}{c}
X_{1}^{+} \\
X_{2}^{+}
\end{array}\right) .
$$

Their masses squared are given by

$$
\begin{aligned}
& m_{1}^{2}=M_{X}^{2}+\frac{v^{2}}{12}\left(6 \lambda_{0}+4 \lambda_{1}-2 \sqrt{\lambda_{1}^{2}+12 \lambda_{2}^{2}}\right), \\
& m_{2}^{2}=M_{X}^{2}+\frac{v^{2}}{12}\left(6 \lambda_{0}+4 \lambda_{1}+2 \sqrt{\lambda_{1}^{2}+12 \lambda_{2}^{2}}\right) .
\end{aligned}
$$

The mass hierarchy of the neutral scalars $\phi$ and $a$ is determined by the sign of $\lambda_{2}$. If $\lambda_{2}>0\left(\lambda_{2}<0\right), \phi$ is lighter (heavier) than $a$, and, thus, $\phi(a)$ is a possible DM candidate. Nevertheless, if $\left|\lambda_{1}\right|>2\left|\lambda_{2}\right|$, one of the singly charged scalars is lighter than the DM candidate. Additionally, if $\lambda_{1}>2\left|\lambda_{2}\right|$, the doubly charged scalar is lighter than the DM candidate. Since the DM candidate should be the lightest particle in the dark sector for ensuring its stability, we have the following conclusions.

(i) If $\lambda_{2}>0$ and $\left|\lambda_{1}\right| \leq 2 \lambda_{2}$, then $\phi$ is a viable DM candidate.

(ii) If $\lambda_{2}<0$ and $\left|\lambda_{1}\right| \leq-2 \lambda_{2}$, then $a$ is a viable DM candidate.

Similar to the IDM, the QSDM model has two kinds of $C P$ symmetries, one with $\phi \rightarrow \phi$ and $a \rightarrow-a$ and the other one with $\phi \rightarrow-\phi$ and $a \rightarrow a$ [59]. A transformation $X^{i j k} \rightarrow i X^{i j k}$ and $\lambda_{2} \rightarrow-\lambda_{2}$ can keep the Lagrangian unchanged but interchange the two $C P$ symmetries and, hence, the roles of $\phi$ and $a$. Therefore, we know that $\phi$ and $a$ have opposite $C P$ parities, but it is impossible to determine their absolute $C P$ parities without additional interactions. Without loss of generality, hereafter we adopt $\lambda_{2}>0$ and take $\phi$ as the DM candidate. The resulting discussions are totally equivalent to those for $\lambda_{2}<0$ and $a$ as the DM candidate.

In the following analyses, four free parameters in the QSDM model are chosen to be $\left\{M_{X}, \lambda_{0}, \lambda_{1}, \lambda_{2}\right\}$. The parameter space is analogous to that of the IDM (cf. Refs. [35-37,59]) in the sense of the number and the roles of the parameters. Nonetheless, the number of dark sector scalars in the QSDM model is more. The IDM dark sector includes two neutral scalars with opposite $C P$ and one singly charged scalar. The neutral scalars in the two models play similar roles, with the lighter one being the DM candidate. On the other hand, the QSDM model contains one more singly charged scalar and an additional doubly charged scalar. After electroweak symmetry breaking, the mass eigenstates of singly charged scalars are different from the gauge eigenstates. This is a new phenomenon that does not exhibit in the IDM.

\section{RELIC ABUNDANCE AND DIRECT DETECTION}

In this section, we evaluate the relic abundance prediction in the QSDM model and investigate the constraints from direct detection experiments.

The dark sector scalars can interact with SM particles via electroweak gauge couplings and scalar couplings to the Higgs boson. Through such interactions, these scalars could be thermally produced in the early Universe and decoupled from the cosmic plasma at the freeze-out epoch. Conventionally, the relic abundance of dark matter is determined by its freeze-out annihilation cross section. Nonetheless, for $m_{X} \sim \mathcal{O}(\mathrm{TeV})$, the mass splittings among the dark sector scalars due to the quartic couplings would be relatively small, and, thus, the scalars actually freeze out around the same epoch. Therefore, the coannihilation effect would be significant for evaluating the relic abundance [60].

There are a lot of relevant annihilation and coannihilation processes. For instance, a $\phi \phi$ pair can annihilate into a SM fermion pair $f \bar{f}$, or an electroweak gauge boson pair $W^{+} W^{-}$or $Z Z$, or a Higgs boson pair $h h$. Some of these annihilation processes are mediated by $s$-channel $Z$ and Higgs bosons, while the others are related to the exchanges of $t$ - and $u$-channel dark sector scalars as well as to the quartic couplings. Because of the significant coannihilation effect, it is not sufficient to just consider the processes that are directly related to DM annihilation. Actually, annihilation or coannihilation between every pair of dark sector scalars could affect the final DM relic abundance.

We utilize a few numerical tools to predict the relic abundance of the DM candidate $\phi$. FeynRules2 [61] is adopted to implement the QSDM model, interfaced to the Monte Carlo generator MADGRAPH5_aMC@NLO2 [62]. The relic abundance $\Omega_{\phi} h^{2}$ is calculated by a MADGRAPH plugin MadDM [63], which can reliably take into account the coannihilation effect. All annihilation and coannihilation diagrams are automatically involved in the calculation.

The measurement of the DM relic abundance in the Planck experiment gives $\Omega_{\mathrm{DM}}=0.1200 \pm 0.0012$ [64]. In Figs. 1(a) and 1(b), we fix the parameters $\left(\lambda_{0}, \lambda_{1}\right)=$ $(0.5,0.05)$ and $\left(\lambda_{0}, \lambda_{2}\right)=(0.7,0.5)$ and show the parameter regions that are consistent with the Planck observation 


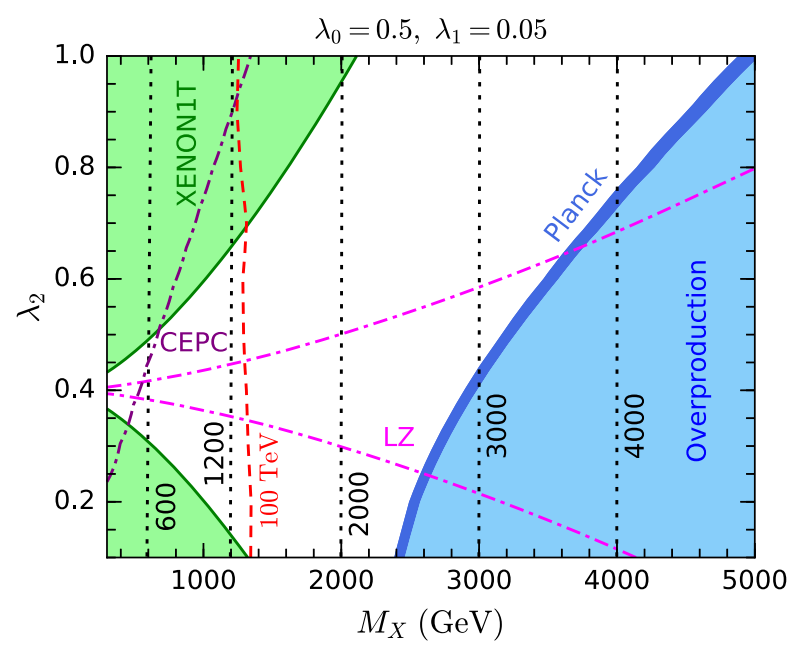

(a) $\lambda_{0}=0.5$ and $\lambda_{1}=0.05$ fixed

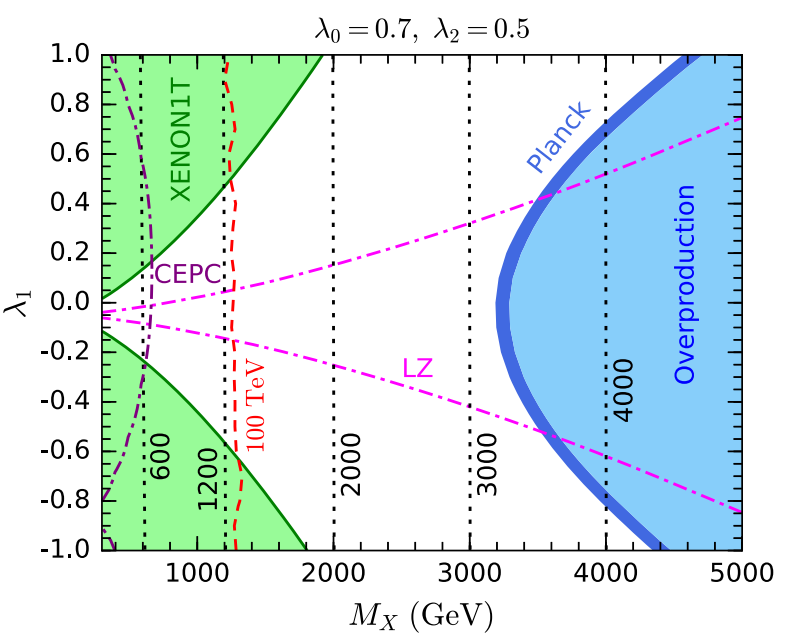

(b) $\lambda_{0}=0.7$ and $\lambda_{2}=0.5$ fixed

FIG. 1. Experimental constraints and sensitivities in the $M_{X}-\lambda_{2}$ (a) and $M_{X}-\lambda_{1}$ (b) planes. The black dotted lines denote the DM candidate mass $m_{\phi}$ in GeV. The blue bands correspond to the $3 \sigma$ range of the Planck relic abundance measurement [64], while the light blue regions predict overproduction of dark matter. The green regions are excluded by the direct detection experiment XENON1T [65]. The dot-dashed magenta lines indicate the exclusion capability of the future direct detect experiment LZ [66]. The dot-dashed purple lines show the expected sensitivities of the measurement of electroweak oblique parameters at the future CEPC. The dashed red lines show the exclusion capability of the monojet $+E_{\mathrm{T}}$ channel at a $100 \mathrm{TeV} p p$ collider with a dataset of $3 \mathrm{ab}^{-1}$ (see Sec. IV).

as the blue bands in the $M_{X}-\lambda_{2}$ and $M_{X}-\lambda_{1}$ planes, respectively. The black dotted lines indicate the contours of the DM candidate mass $m_{\phi}$, which slightly deviates from $M_{X}$ due to the quartic couplings.

If $M_{X}$ increases, the effective annihilation cross section typically decreases, leading to an increase in the relic abundance. Therefore, the light blue regions with large $M_{X}$ predict overproduction of $\phi$ particles in the early Universe, which contradicts standard cosmology. For small values of $\lambda_{2}\left(\left|\lambda_{1}\right|\right)$ in Fig. 1(a) [Fig. 1(b)], the relic abundance observation corresponds to $M_{X} \sim 2.4(3.3) \mathrm{TeV}$, which increases to $M_{X} \sim 5(4.6) \mathrm{TeV}$ when $\lambda_{2}\left(\left|\lambda_{1}\right|\right)$ increases to one. These results are consistent with the simplified calculation given in Ref. [5].

Direct detection experiments look for signals of DM scattering off nuclei. In the QSDM model, DM scattering is mediated by the Higgs boson $h$, arising from the quartic potential terms that lead to the $h \phi \phi$ interaction Lagrangian

$$
\begin{gathered}
\mathcal{L}_{h \phi \phi}=\frac{1}{2} \lambda_{h \phi \phi} v h \phi^{2}, \\
\lambda_{h \phi \phi}=-\lambda_{0}-\frac{2}{3} \lambda_{1}+\frac{4}{3} \lambda_{2} .
\end{gathered}
$$

As direct detection experiments basically operate at zero momentum transfer, the interactions between DM and quarks can be described by dimension- 5 effective operators [67]. As a result, the spin-independent DM-nucleon scattering cross section can be expressed as

$$
\sigma_{\chi N}^{\mathrm{SI}}=\frac{m_{N}^{2} F_{N}^{2}}{4 \pi\left(m_{\phi}+m_{N}\right)^{2}}, \quad N=p, n,
$$

where

$$
F_{N}=-\frac{\lambda_{h \phi \phi} m_{N}}{9 m_{h}^{2}}\left[2+7\left(f_{u}^{N}+f_{d}^{N}+f_{s}^{N}\right)\right]
$$

Here the nucleon form factors $f_{q}^{N}$ are given by [68]

$$
\begin{aligned}
f_{u}^{p} & =0.020 \pm 0.004, \quad f_{d}^{p}=0.026 \pm 0.005 \\
f_{u}^{n} & =0.014 \pm 0.003, \\
f_{d}^{n} & =0.036 \pm 0.008, \quad f_{s}^{p}=f_{s}^{n}=0.118 \pm 0.062
\end{aligned}
$$

In Figs. 1(a) and 1(b), we show the parameter regions excluded by the direct detection experiment XENON1T [65] at 90\% confidence level (C.L.). According to Eq. (17), we can take some particular relations among $\lambda_{0}, \lambda_{1}$, and $\lambda_{2}$ to give a vanishing $h \phi \phi$ coupling, resulting in "blind spots" for direct detection experiments. These relations correspond to the flat directions among the scalar couplings, where the Higgs VEV has zero contribution to $m_{\phi}$. For Figs. 1(a) and 1(b), the limits $\lambda_{2}=3 \lambda_{0} / 4+$ $\lambda_{1} / 2=0.4$ and $\lambda_{1}=-3 \lambda_{0} / 2+2 \lambda_{2}=-0.05$ correspond to $\lambda_{h \phi \phi}=0$, respectively. Therefore, direct detection experiments lose their sensitivities as $\lambda_{2}$ or $\lambda_{1}$ approaches the corresponding limit. Nonetheless, the XENON1T experiment has excluded some disconnected parameter 


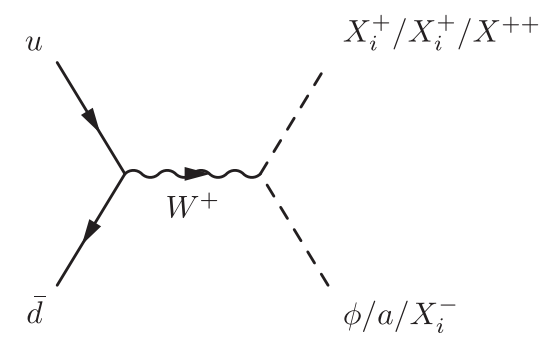

(a)

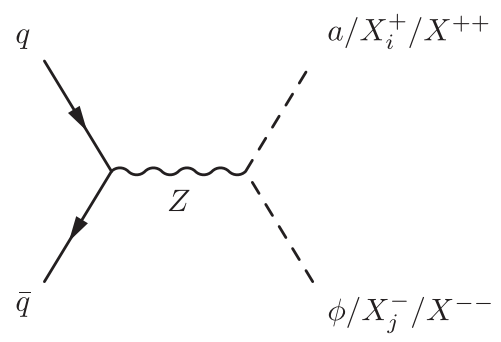

(b)

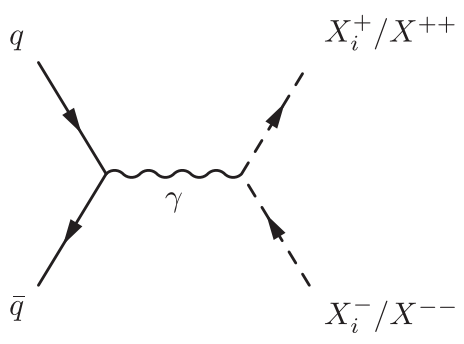

(c)

FIG. 2. Typical diagrams for pair production of dark sector scalars at parton level in $p p$ collisions, including $W^{+}-$mediated $u+\bar{d} \rightarrow$ $X_{i}^{+} / X_{i}^{+} / X^{++}+\phi / a / X_{i}^{-}$(a), Z-mediated $q+\bar{q} \rightarrow a / X_{i}^{+} / X^{++}+\phi / X_{j}^{-} / X^{--}$(b), and $\gamma$-mediated $q+\bar{q} \rightarrow X_{i}^{+} / X^{++}+X_{i}^{-} / X^{--}$(c).

regions with $M_{X} \lesssim 1.3-2 \mathrm{TeV}$. We also demonstrate the expected $90 \%$ C.L. exclusion limits of the future direct detection experiment LZ [66], which will explore the parameter space much deeper and be able to reach the regions suggested by the relic abundance measurement.

Note that the results presented here are based on treelevel calculations. There are also contributions from electroweak loop-induced diagrams [5,12], leading to a nonvanishing spin-independent cross section for the treelevel blind spots. Nevertheless, one would expect a cancellation between the tree and loop diagrams if the scalar couplings are carefully tuned. This means that the blind spots would still exist at loop level, but their positions in the parameter space would be slightly shifted.

As studied in previous papers [10,19], the dark sector scalars in the QSDM model can contribute to the electroweak oblique parameters $S, T$, and $U$ at one-loop level and, hence, affect electroweak precision measurements. In Fig. 1, we also show the $95 \%$ C.L. expected sensitivities of the measurement of electroweak oblique parameters at the future CEPC project [43]. This result is estimated following the strategy in our previous work [19] with the optimistic settings. We can see that the CEPC experiment

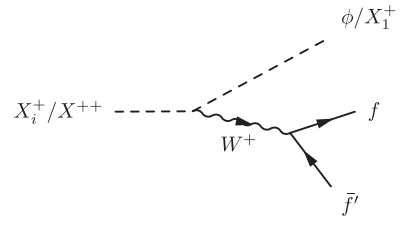

(a)

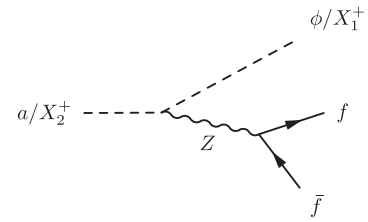

(c)

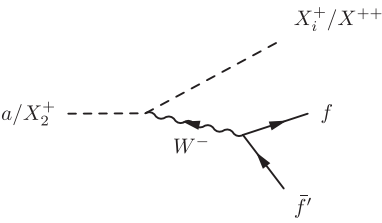

(b)

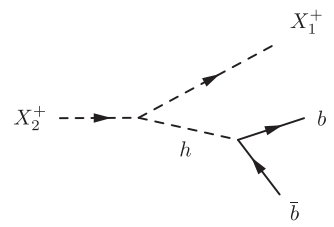

(d)
FIG. 3. Typical decay diagrams for dark sector scalars, including $X_{i}^{+} / X^{++} \rightarrow W^{+(*)}+\phi / X_{1}^{+}$(a), $a / X_{2}^{+} \rightarrow W^{-(*)}+X_{i}^{+} / X^{++}$ (b), $a / X_{2}^{+} \rightarrow Z^{(*)}+\phi / X_{1}^{+}$(c), and $X_{2}^{+} \rightarrow h^{(*)}+X_{1}^{+}$(d). would probe up to $m_{X} \sim 600-1200 \mathrm{GeV}$, covering some regions related to the blind spots in direct detection.

\section{MONOJET SEARCHES AT $p p$ COLLIDERS}

Through the electroweak gauge couplings, the dark sector scalars in the QSDM model could be directly produced in pairs at the LHC. The corresponding processes can be expressed as $p p \rightarrow \chi_{i} \chi_{j}+$ jets with $\chi_{i}=\left(\phi, a, X_{1}^{ \pm}, X_{2}^{ \pm}, X^{ \pm \pm}\right)$. Figure 2 shows some typical parton-level diagrams for pair production of dark sector scalars at the LHC. After production, a heavier scalar $\chi_{k}$ may decay into a lighter scalar $\chi_{l}$ via $\chi_{k} \rightarrow W^{ \pm(*)} /$ $Z^{(*)} / h^{(*)}+\chi_{l}$. Typical decay diagrams are demonstrated in Fig. 3. Depending on the mass splitting between $\chi_{k}$ and $\chi_{l}$, the produced $W^{ \pm}, Z$, and $h$ bosons can be either on or off shell. Subsequent decays may happen and form decay chains. Finally, all $Z_{2}$-odd scalars will decay into the DM candidate $\phi$, which is stable and escapes from detection, leading to a large $\mathbb{E}_{\mathrm{T}}$.

Figure 4 shows the mass splittings between $\left(a, X_{1}^{ \pm}\right.$, $\left.X_{2}^{ \pm}, X^{ \pm \pm}\right)$and $\phi$ as functions of $M_{X}$ for $\lambda_{0}=0.1$ and

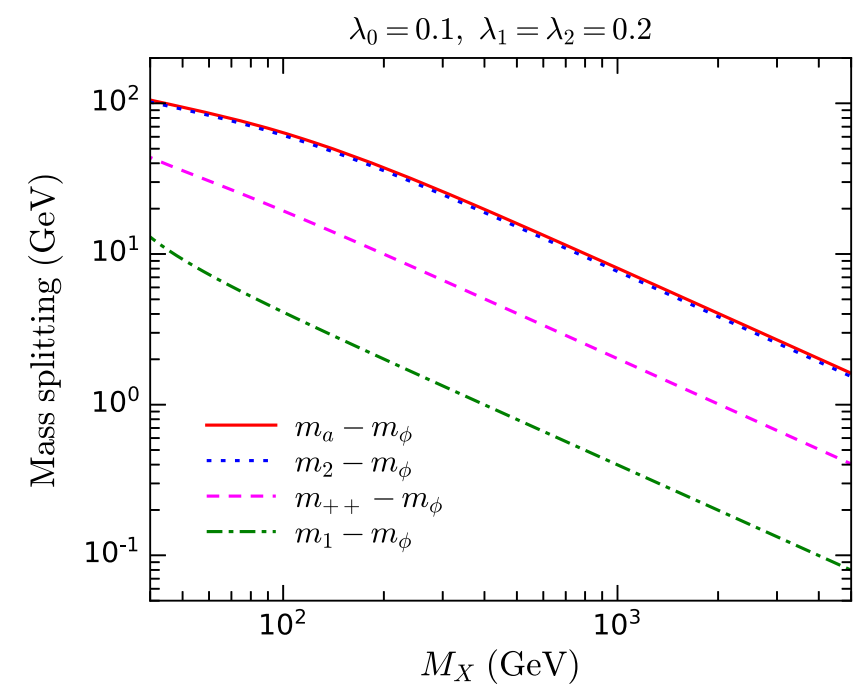

FIG. 4. Mass splittings as functions of $M_{X}$ for $\lambda_{0}=0.1$ and $\lambda_{1}=\lambda_{2}=0.2$. 
TABLE I. Reconstruction and cut conditions in the monojet + $E_{\mathrm{T}}$ channel.

\begin{tabular}{lcc}
\hline \hline & $13 \mathrm{TeV}$ LHC & $100 \mathrm{TeV} p p$ collider \\
\hline \multicolumn{3}{c}{ Reconstruction conditions } \\
Electron $p_{\mathrm{T}},|\eta|$ & $>20 \mathrm{GeV},<2.47$ & $>40 \mathrm{GeV},<2.47$ \\
Muon $p_{\mathrm{T}},|\eta|$ & $>10 \mathrm{GeV},<2.5$ & $>20 \mathrm{GeV},<2.5$ \\
Jet $p_{\mathrm{T}},|\eta|$ & $>30 \mathrm{GeV},<2.8$ & $>60 \mathrm{GeV},<2.8$ \\
\multicolumn{4}{c}{ Cut conditions } & 0 \\
Number of leptons & 0 & $>1.4 \mathrm{TeV},<2.4$ \\
Leading jet $p_{\mathrm{T}},|\eta|$ & $>250 \mathrm{GeV},<2.4$ & $\leq 4$ \\
Number of jets & $\leq 4$ & $>0.4$ \\
$\Delta \phi\left(j_{i}, \not p_{\mathrm{T}}\right)$ & $>0.4$ & $>1.5-2.8 \mathrm{TeV}$ \\
$E_{\mathrm{T}}$ & $>250-1000 \mathrm{GeV}$ &
\end{tabular}

$\lambda_{1}=\lambda_{2}=0.2$. From this plot, we can read off the mass difference between each pair of dark sector scalars. As $M_{X}$ increases, the contributions from the quartic couplings relatively decrease, resulting in smaller splittings. The mass splitting between the two neutral scalars $a$ and $\phi$ is the largest one, ranging from $\sim 100$ to $\sim 2 \mathrm{GeV}$ as $m_{X}$ increases from $40 \mathrm{GeV}$ to $5 \mathrm{TeV}$. For $m_{X} \gtrsim 70 \mathrm{GeV}$, the splittings are not large enough to induce on-shell $W^{ \pm}, Z$, or $h$ bosons. For fixed $M_{X}$, smaller quartic couplings would further compress the mass spectrum.

In the above analysis, we find that the mass splittings in the QSDM model are typically small. Consequently, visible decay products from the dark sector scalars would be quite soft and, hence, difficult to be triggered in detectors. In order to effectively trigger the signal, we can require at least one hard jet from initial state radiation to recoil the $\chi_{i} \chi_{j}$ pair, leading to a monojet $+\mathbb{E}_{\mathrm{T}}$ final state [44-46]. SM backgrounds in the monojet $+\mathbb{E}_{\mathrm{T}}$ search channel include two major backgrounds- $W(\rightarrow l \nu)+$ jets and $Z(\rightarrow \nu \bar{\nu})+$ jets - and some minor backgrounds, such as $t \bar{t}+$ jets and $V V+$ jets $\left(V=W^{ \pm}, Z\right)$. In these backgrounds, $\mathbb{E}_{\mathrm{T}}$ mainly arises from neutrinos in the decay products.

\section{A. LHC constraint}

In this subsection, we investigate the current LHC constraint on the QSDM model by reinterpreting the ATLAS analysis in the monojet $+\mathbb{E}_{\mathrm{T}}$ channel with an integrated luminosity of $36.1 \mathrm{fb}^{-1}$ at $\sqrt{s}=13 \mathrm{TeV}$ [69]. For this purpose, we utilize MADGRAPH [62] to generate signal simulation samples. Parton shower is performed by PYTHIA8 [70] with the MLM matching scheme [71]. PYTHIA is also carried out for hadronization and decay processes. Then we use DELPHES3 [72] for a fast detector simulation with a setup for the ATLAS detector.

We simulate the signal processes $p p \rightarrow \chi_{i} \chi_{j}+$ jets and apply the same selection cuts in the ATLAS analysis [69] to the simulation events. Isolated leptons, including electrons and muons, and jets are reconstructed with the conditions on $p_{\mathrm{T}}$ and $\eta$ listed in Table I. Then the events in the signal regions are required to have a hard leading jet with $p_{\mathrm{T}}>$ $250 \mathrm{GeV}$ and $|\eta|<2.4$ and a missing transverse energy $\mathbb{E}_{\mathrm{T}}$ at least larger than $250 \mathrm{GeV}$. In addition, there should be no leptons and no more than four jets. Moreover, the separation in the azimuthal angle between any reconstructed jet $j_{i}$ and the missing transverse momentum $\not \not_{\mathrm{T}}$ should satisfy $\Delta \phi\left(j_{i}, \not \not_{\mathrm{T}}\right)>0.4$ for preventing a large $\boldsymbol{E}_{\mathrm{T}}$ from mismeasurement of jets. Finally, ten inclusive and ten exclusive signal regions are defined with different $\mathbb{E}_{\mathrm{T}}$ thresholds, whose explicit definitions can be found in Table 1 of Ref. [69]. In Table I, we summarize the cut conditions above.

Based on the signal simulation samples, we estimate the visible cross section in each signal region, which is a product of the production cross section, acceptance, and efficiency, and then use the 95\% C.L. observed experimental upper limit to derive constraints on the QSDM model. Taking into account all the signal regions, the combined exclusion region in the $m_{\phi}-\lambda_{2}$ plane is shown in Fig. 5(a), where we fix a coupling relation of $\lambda_{1}=$ $\lambda_{2}=3 \lambda_{0} / 2$. Because of Eq. (17), such a relation leads to $\lambda_{h \phi \phi}=0$, and there is no constraint from direct detection experiments. Therefore, collider searches are really important in this case. Note that $\lambda_{h \phi \phi}=0$ also leads to $m_{\phi}=m_{X}$, according to Eqs. (6) and (17). We find that the monojet search has excluded a region with $m_{\phi} \lesssim 33 \mathrm{GeV}$ and $\lambda_{2} \lesssim 0.3$. Nonetheless, the sensitivity decreases as $\lambda_{2}$ increases. The reason is that a larger $\lambda_{2}$ leads to larger mass splittings among the dark sector scalars and, hence, harder leptons from scalar decays that would not be easy to pass the cuts.

In Fig. 5(b), we adopt another relation $\lambda_{0}=0$ and $\lambda_{1}=2 \lambda_{2}$, which also results in $\lambda_{h \phi \phi}=0$. Additionally, it leads to degenerate mass spectra with $m_{\phi}=m_{1}=m_{++}$ and $m_{a}=m_{2}$. Consequently, many decay channels are turned off, significantly reducing the probability of finding leptons in the final state. Therefore, the monojet search is more sensitive, excluding a region up to $m_{\phi} \sim 45 \mathrm{GeV}$. The exclusion is basically regardless of the $\lambda_{2}$ value.

\section{B. Sensitivity at a $100 \mathrm{TeV} p p$ collider}

The above results have shown that the current LHC monojet search is rather insensitive to the QSDM model, just probing a scale of a few tens of GeV. Since production cross sections in $p p$ collisions typically increase as $\sqrt{s}$ increases, we expect that monojet searches at a future $p p$ collider with $\sqrt{s} \sim 100 \mathrm{TeV}$ would be much more sensitive. Below, we estimate the projected sensitivity in the monojet $+\mathbb{E}_{\mathrm{T}}$ channel at a $100 \mathrm{TeV} p p$ collider based on simulation. The obtained results would be applicable to both the SPPC and FCC-hh projects.

In the simulation with $\sqrt{s}=100 \mathrm{TeV}$, we consider the signal processes and only the primary SM backgrounds $W(\rightarrow \ell \nu)+$ jets and $Z(\rightarrow \nu \bar{\nu})+$ jets. Other backgrounds 


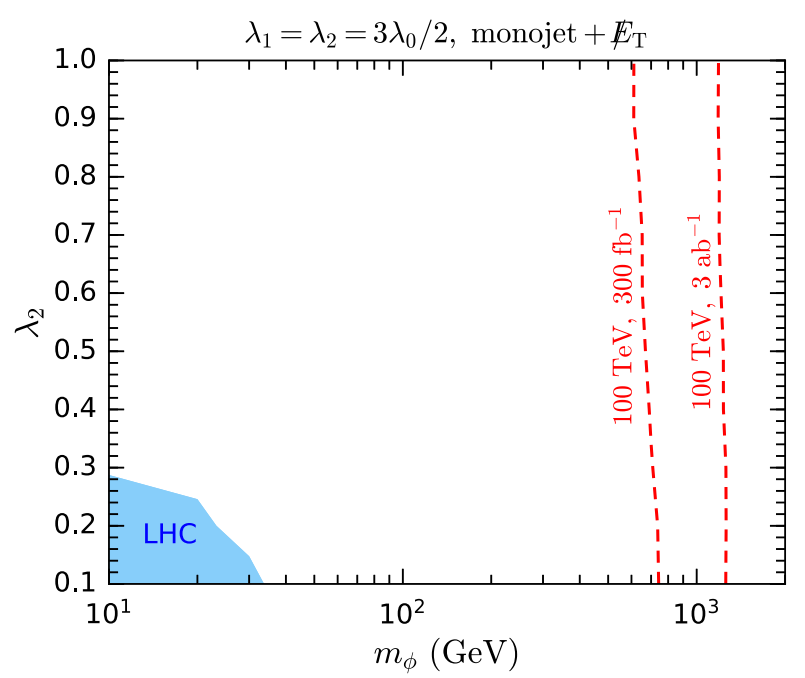

(a)

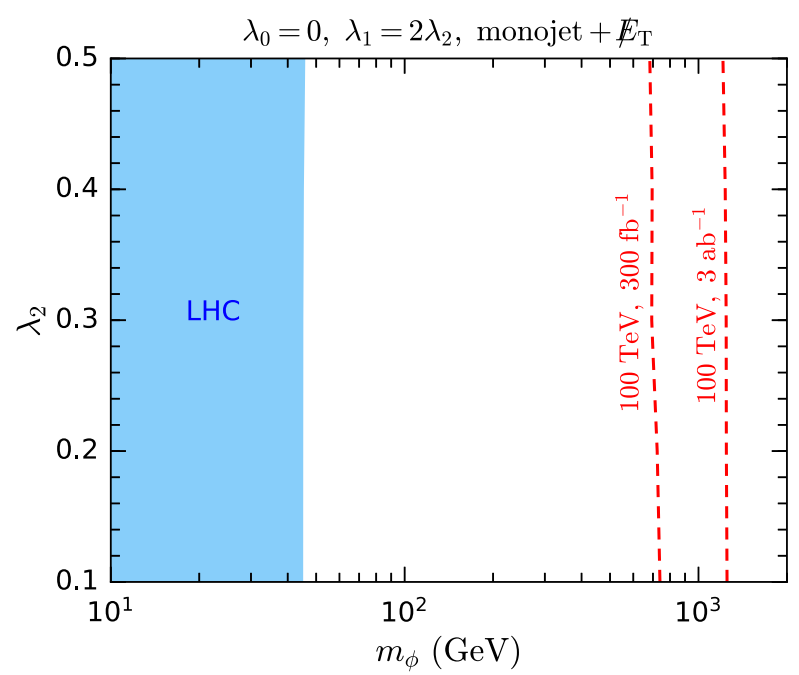

(b)

FIG. 5. Current constraints and future sensitivities from the monojet $+\mathscr{E}_{\mathrm{T}}$ channel in the $m_{\phi}-\lambda_{2}$ plane for the fixed coupling relations of $\lambda_{1}=\lambda_{2}=3 \lambda_{0} / 2$ (a) and of $\lambda_{0}=0$ and $\lambda_{1}=2 \lambda_{2}$ (b). The blue regions are excluded at 95\% C.L. by the ATLAS search with a $36.1 \mathrm{fb}^{-1}$ dataset at the $13 \mathrm{TeV}$ LHC [69]. The red dashed lines denote the 95\% C.L. expected exclusion limits at a $100 \mathrm{TeV} p p$ collider with integrated luminosities of $300 \mathrm{fb}^{-1}$ and $3 \mathrm{ab}^{-1}$.

should be small and can be safely neglected. In the DELPHES simulation, we conservatively assume that the future detector has the same parameters as those in the ATLAS detector. The thresholds in the reconstruction and cut conditions are appropriately adjusted for a $100 \mathrm{TeV}$ $p p$ collider, as also demonstrated in Table I.

Four signal regions are defined by requiring $\mathbb{E}_{\mathrm{T}}>1.5$, $1.8,2.2,2.8 \mathrm{TeV}$. In each signal region, the signal significance $\mathcal{S}$ is defined as

$$
\mathcal{S}=\frac{S}{\sqrt{S+B}}
$$

where $S$ and $B$ are the estimated numbers of the signal events and the total background events passing the corresponding cuts, respectively.

In order to show the cut efficiency in the monojet $+\mathbb{E}_{\mathrm{T}}$ channel, we adopt four benchmark points (BMPs) for the QSDM model, whose parameters and mass spectra are listed in Table II. All of them satisfy $\lambda_{1}=\lambda_{2}=3 \lambda / 2$, leading to vanishing $h \phi \phi$ coupling and $m_{\phi}=m_{X}$. Thus, they would not be constrained by direct detection. We choose the same $\lambda_{2}$ but different $m_{X}$ for the four BMPs. As discussed above, a larger $m_{X}$ leads to a more compressed mass spectrum. The predicted relic abundances of these BMPs are lower than the observed value.

For the signal region with $\mathscr{E}_{\mathrm{T}}>1.5 \mathrm{TeV}$, we divide the cut conditions into the following four cuts.

(i) Cut 1.- At least one reconstructed jet, and the leading jet with $p_{\mathrm{T}}>1.4 \mathrm{TeV}$ and $|\eta|<2.4$.

(ii) Cut 2.-No reconstructed lepton.

(iii) $\mathrm{Cut}$ 3.-At most four reconstructed jets, and $\Delta \phi\left(j_{i}, \not p_{\mathrm{T}}\right)>0.4$.

(iv) Cut 4. $-\mathbb{E}_{\mathrm{T}}>1.5 \mathrm{TeV}$.

After applying the cuts one by one, the visible cross section $\sigma_{\text {vis }}$ and the signal significance $\mathcal{S}$ for an integrated luminosity of $3 \mathrm{ab}^{-1}$ are tabulated in Table III. While cut 2 does not affect the $Z(\rightarrow \nu \bar{\nu})+$ jets background, it reduces the $W(\rightarrow \ell \nu)+$ jets background which has a genuine lepton in the final state. Cut 3 and cut 4 combined suppress the $W(\rightarrow \ell \nu)+$ jets $[Z(\rightarrow \nu \bar{\nu})+$ jets] background by 2 (1) orders of magnitude. For all the BMPs, these cuts subsequently increase the signal significance. Note that Eq. (21) does not take into account systematic

TABLE II. Information of the four benchmark points with the fixed coupling relation $\lambda_{1}=\lambda_{2}=3 \lambda_{0} / 2$ for the monojet $+\mathscr{E}_{\mathrm{T}}$ channel.

\begin{tabular}{lccccrrr}
\hline \hline & $M_{X} / \mathrm{GeV}$ & $\lambda_{2}$ & $m_{++} / \mathrm{GeV}$ & $m_{\phi} / \mathrm{GeV}$ & $m_{a} / \mathrm{GeV}$ & $m_{1} / \mathrm{GeV}$ & $m_{2} / \mathrm{GeV}$ \\
\hline BMP-a & 400 & 0.2 & 405.0 & 400 & 419.7 & 401.0 & 418.7 \\
BMP-b & 700 & 0.2 & 702.9 & 700 & 711.4 & 700.6 & 710.9 \\
BMP-c & 1000 & 0.2 & 1002.0 & 1000 & 1008.0 & 1000.4 & 1007.6 \\
BMP-d & 1300 & 0.2 & 1301.6 & 1300 & 1306.2 & 1300.3 & 1305.9 \\
\hline \hline
\end{tabular}


TABLE III. Visible cross section $\sigma_{\text {vis }}$ in femtobarns and signal significance $\mathcal{S}$ for integrated luminosity $3 \mathrm{ab}^{-1}$ after each cut in the signal region with $\mathscr{E}_{\mathrm{T}}>1.5 \mathrm{TeV}$ of the monojet $+\mathscr{E}_{\mathrm{T}}$ channel at $\sqrt{s}=100 \mathrm{TeV}$.

\begin{tabular}{|c|c|c|c|c|c|c|c|c|c|c|}
\hline & \multirow{2}{*}{$\frac{W \rightarrow \ell \nu}{\sigma_{\mathrm{vis}}}$} & \multirow{2}{*}{$\frac{Z \rightarrow \nu \bar{\nu}}{\sigma_{\text {vis }}}$} & \multicolumn{2}{|c|}{ BMP-a } & \multicolumn{2}{|c|}{ BMP-b } & \multicolumn{2}{|c|}{ BMP-c } & \multicolumn{2}{|c|}{ BMP-d } \\
\hline & & & $\sigma_{\mathrm{vis}}$ & $\mathcal{S}$ & $\sigma_{\mathrm{vis}}$ & $\mathcal{S}$ & $\sigma_{\mathrm{vis}}$ & $\mathcal{S}$ & $\sigma_{\text {vis }}$ & $\mathcal{S}$ \\
\hline Cut 1 & 6080 & 1481 & 8.08 & 5.08 & 3.16 & 1.99 & 1.41 & 0.89 & 0.73 & 0.46 \\
\hline Cut 2 & 4428 & 1481 & 7.79 & 5.54 & 3.11 & 2.21 & 1.41 & 1.00 & 0.73 & 0.52 \\
\hline Cut 3 & 1442 & 654 & 5.24 & 6.26 & 2.32 & 2.77 & 1.07 & 1.27 & 0.56 & 0.66 \\
\hline Cut 4 & 62.7 & 139 & 3.65 & 13.8 & 1.80 & 6.81 & 0.87 & 3.31 & 0.47 & 1.79 \\
\hline
\end{tabular}

uncertainties, which could be a few to ten percent in monojet searches. If systematic uncertainties are considered, the signal significance would be reduced. Since BMPb, -c, and -d have a $S / B$ ratio below $1 \%$, it could be difficult to test them in this signal region.

Combining the four signal regions, the expected exclusion limits at 95\% C.L. are shown in Figs. 5(a) and 5(b). For datasets of $300 \mathrm{fb}^{-1}$ and $3 \mathrm{ab}^{-1}$ at $\sqrt{s}=100 \mathrm{TeV}$, monojet searches are expected to probe the DM candidate mass $m_{\phi}$ up to $\sim 700 \mathrm{GeV}$ and $\sim 1.2 \mathrm{TeV}$, respectively. Thus, a $100 \mathrm{TeV} p p$ collider looks much more powerful than the LHC.

In order to compare with direct detection and relic abundance observation, we have also plotted the 95\% C.L. expected exclusion limits in the monojet $+\mathbb{E}_{\mathrm{T}}$ channel at $\sqrt{s}=100 \mathrm{TeV}$ with an integrated luminosity of $3 \mathrm{ab}^{-1}$ in Figs. 1(a) and 1(b). We find that the $100 \mathrm{TeV}$ monojet searches could cover some regions where direct detection experiments cannot probe. Nonetheless, the regions predicting an observed relic abundance could not be reached.

\section{SOFT-LEPTON SEARCHES AT $p p$ COLLIDERS}

Besides the monojet channel, leptons arising from the scalar decays $\chi_{i} \rightarrow \chi_{j}+W^{ \pm(*)}\left(\rightarrow \ell^{ \pm} \nu_{\ell}\right) / Z^{(*)}\left(\rightarrow \ell^{ \pm} \ell^{\mp}\right)$ may also contain important information for exploring the QSDM model. Inspired by the searches for electroweak production of charginos and neutralinos in supersymmetric models, we first consider the final states involving two or three "hard" leptons. After recasting the related ATLAS analysis at $\sqrt{s}=13 \mathrm{TeV}$ with a dataset of $36.1 \mathrm{fb}^{-1}$ [73], however, we do not find any meaningful constraint on the QSDM model. The main reason is that the leptons from the scalar decays tend to be rather soft, because the mass spectrum is typically compressed, as explained in the previous section.

Therefore, it is more suitable to consider the final states with "soft" leptons. In this case, a pair of same-flavor opposite-sign (SFOS) soft leptons with an invariant mass $\lesssim 60 \mathrm{GeV}$ could lead to a distinct signature [53,54]. In the signal process $p p \rightarrow \chi_{i} \chi_{j}+$ jets, such a SFOS lepton pair may come from the scalar decays into an off-shell $Z$ boson. In order to induce a sufficiently large $\mathbb{E}_{\mathrm{T}}$, a hard jet with a transverse direction roughly opposite to that of $\not p_{\mathrm{T}}$ is also required. Such a soft - leptons + jets $+\mathscr{E}_{\mathrm{T}}$ channel has been utilized in the ATLAS search for supersymmetric particles with compressed mass spectra at the $13 \mathrm{TeV}$ LHC with an integrated luminosity of $36.1 \mathrm{fb}^{-1}$ [74]. Important SM backgrounds in this channel include $t \bar{t}+$ jets, $t W+$ jets, $V V+$ jets, and $\tau^{+} \tau^{-}+$jets.

\section{A. $\mathrm{LHC}$ constraint}

We reinterpret the ATLAS analysis [74] to study the current constraint on the QSDM model in the soft leptons + jets $+\mathbb{E}_{\mathrm{T}}$ channel. The corresponding reconstruction and cut conditions are summarized in Table IV. The $p_{\mathrm{T}}$ thresholds for reconstructed electrons and muons are lowered to 4.5 and $4 \mathrm{GeV}$ for keeping soft leptons in the final state. There should be exact two leptons forming a SFOS pair, whose direction distance $\Delta R_{\ell \ell}$ and invariant mass $m_{\ell \ell}$ should lie in proper ranges because they are considered to be originated from an off-shell $Z$ boson. Events with $m_{\ell \ell} \in(3,3.2) \mathrm{GeV}$ are rejected to avoid

TABLE IV. Reconstruction and cut conditions in the ATLAS soft-leptons + jets $+\mathscr{E}_{\mathrm{T}}$ analysis at $\sqrt{s}=13 \mathrm{TeV}[74]$.

\begin{tabular}{|c|c|}
\hline \multicolumn{2}{|c|}{ Reconstruction conditions } \\
\hline Electron $p_{\mathrm{T}},|\eta|$ & $>4.5 \mathrm{GeV},<2.47$ \\
\hline Muon $p_{\mathrm{T}},|\eta|$ & $>4 \mathrm{GeV},<2.5$ \\
\hline Non- $b$-tagged jet $p_{\mathrm{T}},|\eta|$ & $>30 \mathrm{GeV},<2.8$ \\
\hline$b$-tagged jet $p_{\mathrm{T}},|\eta|$ & $>20 \mathrm{GeV},<2.5$ \\
\hline \multicolumn{2}{|c|}{ Cut conditions } \\
\hline Number of leptons & 2 \\
\hline Lepton flavor and charge & $e^{+} e^{-}$or $\mu^{+} \mu^{-}$ \\
\hline Leading lepton $p_{\mathrm{T}}^{\ell_{1}}$ & $>5 \mathrm{GeV}$ \\
\hline Subleading lepton $p_{\mathrm{T}}^{\ell_{2}}$ & $>4.5(4) \mathrm{GeV}$ for $\ell_{2}=e(\mu)$ \\
\hline$\Delta R_{\ell \ell}$ & $0.05<\Delta R_{\ell \ell}<2$ \\
\hline$m_{\ell \ell}$ & {$[1,3] \cup[3.2,60] \mathrm{GeV}$} \\
\hline$E_{\mathrm{T}}$ & $>200 \mathrm{GeV}$ \\
\hline Number of jets & $\geq 1$ \\
\hline Leading jet $p_{\mathrm{T}}$ & $>100 \mathrm{GeV}$ \\
\hline$\Delta \phi\left(j_{1}, \not \phi_{\mathrm{T}}\right)$ & $>2$ \\
\hline $\min \left(\Delta \phi\left(j_{i}, \not \not_{\mathrm{T}}\right)\right)$ & $>0.4$ \\
\hline Number of $b$-tagged jets & 0 \\
\hline$m_{\tau \tau}$ & $<0$ or $>160 \mathrm{GeV}$ \\
\hline$m_{\mathrm{T}}^{\ell_{1}}$ & $<70 \mathrm{GeV}$ \\
\hline $\mathscr{E}_{\mathrm{T}} / H_{\mathrm{T}}^{\mathrm{lep}}$ & $>\max \left(5,15-2 m_{\ell \ell} / \mathrm{GeV}\right)$ \\
\hline
\end{tabular}


TABLE V. Signal regions defined by the $m_{\ell \ell}$ bins in the ATLAS soft-leptons + jets $+\boldsymbol{E}_{\mathrm{T}}$ analysis at $\sqrt{s}=13 \mathrm{TeV}$ [74].

\begin{tabular}{llllllll}
\hline Signal regions & SR1 & SR2 & SR3 & SR4 & SR5 & SR6 & SR7
\end{tabular}

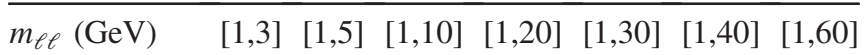

$\begin{array}{llllllll}\sigma_{\text {vis }}^{\text {obs }}(\mathrm{fb}) & 0.10 & 0.18 & 0.34 & 0.61 & 0.59 & 0.72 & 0.80\end{array}$

contamination from $J / \psi$ decays. In order to increase the signal-to-background ratio, at least one jet with $p_{\mathrm{T}}>$ $100 \mathrm{GeV}$ and $E_{\mathrm{T}}>200 \mathrm{GeV}$ are required. The condition $\Delta \phi\left(j_{1}, \not \not_{\mathrm{T}}\right)>2$ is used to ensure the transverse directions of the leading jet and $\not \not_{\mathrm{T}}$ are quite opposite. In order to suppress the $t \bar{t}+$ jets and $t W+$ jets backgrounds, no $b$-tagged jet is allowed.

For further increasing the signal significance, some dedicated kinematic variables are utilized. The $m_{\tau \tau}$ variable $[53,54,74,75]$ constructed by the SFOS lepton pair is helpful for reducing the $\tau^{+} \tau^{-}+$jets background. The leading lepton transverse mass $m_{\mathrm{T}}^{\ell_{1}}=$ $\sqrt{2\left(E_{\mathrm{T}}^{\ell_{1}} \boldsymbol{E}_{\mathrm{T}}-\mathbf{p}_{\mathrm{T}}^{\ell_{1}} \cdot \not p_{\mathrm{T}}\right)}$ is required to satisfy $m_{\mathrm{T}}^{\ell_{1}}<$ $70 \mathrm{GeV}$, in order to suppress the $t \bar{t}+$ jets, $V V+$ jets, and $W+$ jets backgrounds. The ratio of $\mathbb{E}_{\mathrm{T}}$ to the scalar sum of the transverse momenta of the leptons $H_{\mathrm{T}}^{\text {lep }}=$ $p_{\mathrm{T}}^{\ell_{1}}+p_{\mathrm{T}}^{\ell_{2}}$ is used to improve the signal-to-background discrimination for compressed spectra.

We find that seven signal regions in the ATLAS analysis [74] could be sensitive to the QSDM model. They are defined with different inclusive $m_{\ell \ell}$ bins, as tabulated in Table V. Note that the $(3,3.2) \mathrm{GeV}$ interval has also removed in these bins. We also list the corresponding

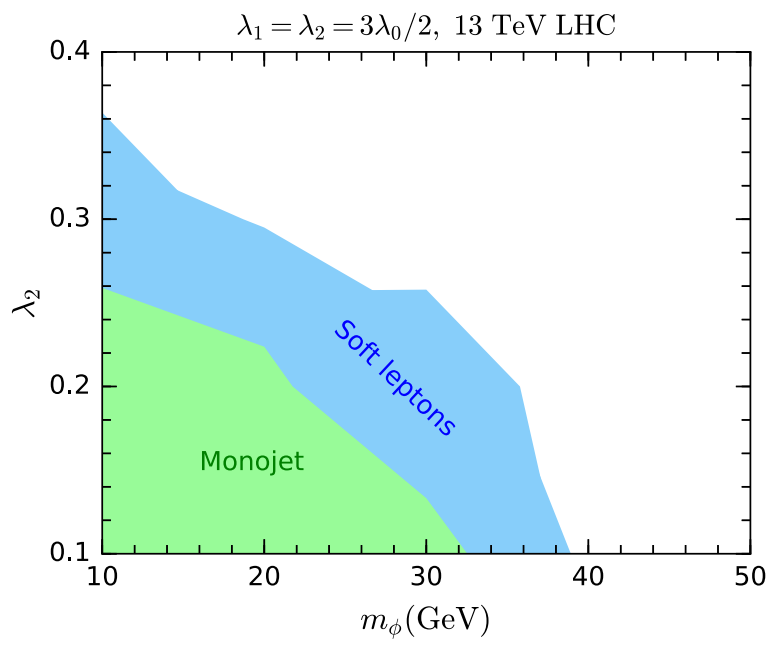

(a) $\lambda_{2} \geq 0.1$
95\% C.L. observed limits on the visible cross section, $\sigma_{\mathrm{vis}}^{\mathrm{obs}}$. We thus simulate signal samples and apply the above cuts to obtain 95\% C.L. exclusion limits on the QSDM model.

The exclusion regions from the seven signal regions are combined, shown as the blue regions in Fig. 6. The fixed coupling relation in Figs. 6(a) and 6(b) is identical to that in Fig. 5(a). For comparison, we also demonstrate the green regions excluded by the ATLAS monojet search, which has been discussed in the previous section. We find that the soft-leptons + jets $+E_{\mathrm{T}}$ channel is more sensitive than the monojet $+\mathbb{E}_{\mathrm{T}}$ channel at $\sqrt{s}=13 \mathrm{TeV}$. In Fig. 6(a) for $\lambda_{2} \geq 0.1$, the soft-lepton search has excluded a region with $m_{\phi} \lesssim 39 \mathrm{GeV}$. In Fig. 6(b), we focus on the small $\lambda_{2}$ region $\left(\lambda_{2} \leq 0.09\right)$ and find that the soft-lepton search can probe up to $m_{\phi} \sim 85 \mathrm{GeV}$ for $\lambda_{2} \lesssim 0.03$. The reason is that the $E_{\mathrm{T}} / H_{\mathrm{T}}^{\text {lep }}$ cut is more suitable for small mass splittings, say, $m_{a}-m_{\phi} \lesssim 20 \mathrm{GeV}$, which is realized in such a $\lambda_{2} \lesssim 0.03$ region.

\section{B. Sensitivity at a $100 \mathrm{TeV} p p$ collider}

In this subsection, we explore the soft-leptons + jets + $\mathscr{E}_{\mathrm{T}}$ channel at a $100 \mathrm{TeV} p p$ collider. The main backgrounds $t \bar{t}+$ jets, $t W+$ jets, $V V+$ jets, and $\tau^{+} \tau^{-}+$jets are taken into account. In order to demonstrate a detailed study, we choose four BMPs for this channel with the fixed coupling relation $\lambda_{1}=\lambda_{2}=3 \lambda_{0} / 2$, which leads to $\lambda_{h \phi \phi}=$ 0 and $m_{\phi}=m_{X}$. The parameters and mass spectra of the BMPs are displayed in Table VI. These BMPs would not be constrained by direct detection experiments, and they predict DM relic abundance lower than the observation. BMP1 and BMP2 have identical $M_{X}$ and different $\lambda_{2}$, and, thus, a large $\lambda_{2}$ leads to larger mass splittings. If $\lambda_{2}$ is fixed,

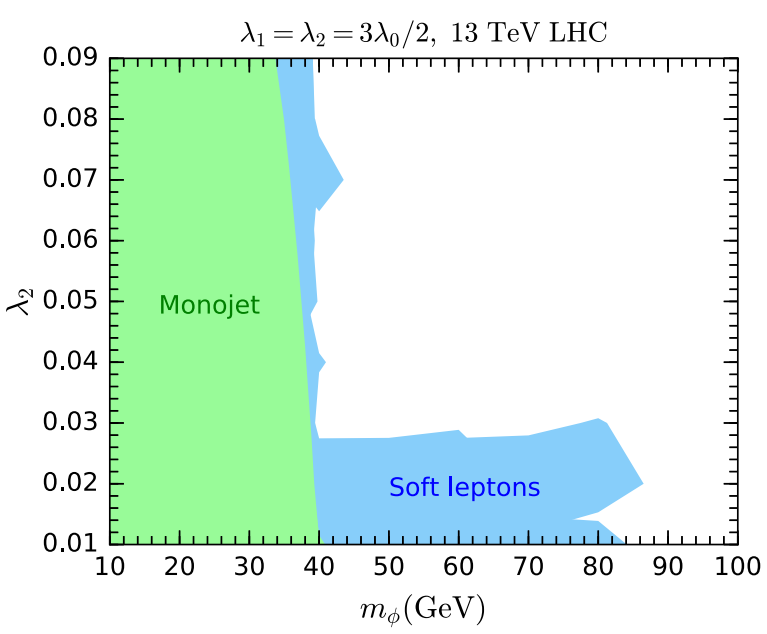

(b) $\lambda_{2} \leq 0.09$

FIG. 6. Constraints from current LHC searches in the $m_{\phi}-\lambda_{2}$ plane with the fixed coupling relation $\lambda_{1}=\lambda_{2}=3 \lambda_{0} / 2$ for $\lambda_{2} \geq 0.1$ (a) and $\lambda_{2} \leq 0.09$ (b). Blue (green) regions are excluded at 95\% C.L. by the ATLAS soft-leptons + jets $+\mathbb{E}_{\mathrm{T}}\left[\right.$ [74] (monojet $+\not \mathbb{E}_{\mathrm{T}}[69]$ ) analysis at the $13 \mathrm{TeV}$ LHC with a dataset of $36.1 \mathrm{fb}^{-1}$. 
TABLE VI. Information of the four benchmark points with the fixed coupling relation $\lambda_{1}=\lambda_{2}=3 \lambda_{0} / 2$ for the soft-leptons + jets $+\mathbb{E}_{\mathrm{T}}$ channel.

\begin{tabular}{lccccccc}
\hline \hline & $M_{X} / \mathrm{GeV}$ & $\lambda_{2}$ & $m_{++} / \mathrm{GeV}$ & $m_{\phi} / \mathrm{GeV}$ & $m_{a} / \mathrm{GeV}$ & $m_{1} / \mathrm{GeV}$ & $m_{2} / \mathrm{GeV}$ \\
\hline BMP1 & 400 & 0.2 & 405.0 & 400 & 419.7 & 401.0 & 418.7 \\
BMP2 & 400 & 0.4 & 410.0 & 400 & 438.5 & 402.0 & 436.7 \\
BMP3 & 500 & 0.2 & 504.0 & 500 & 515.9 & 500.8 & 515.1 \\
BMP4 & 200 & 0.4 & 219.2 & 200 & 268.8 & 203.9 & 265.9 \\
\hline \hline
\end{tabular}

TABLE VII. Cut conditions in the soft-leptons + jets $+\mathscr{E}_{\mathrm{T}}$ channel at a $100 \mathrm{TeV} p p$ collider.

\begin{tabular}{cc}
\hline \hline Cut 1 & Exact two SFOS leptons \\
& Leading lepton $p_{\mathrm{T}}>12 \mathrm{GeV}, 0.05<\Delta R_{\ell \ell}<2$ \\
Cut 2 & At least one jet, no $b$-tagged jet \\
& Leading jet $p_{\mathrm{T}}>200 \mathrm{GeV}$ \\
Cut 3 & $\Delta \phi\left(j_{1}, \not \phi_{\mathrm{T}}\right)>2.0, \min \left(\Delta \phi\left(j_{i}, \not \not_{\mathrm{T}}\right)\right)>0.4$ \\
Cut 4 & $m_{\tau \tau}<0$ or $m_{\tau \tau}>200 \mathrm{GeV}$ \\
Cut 5 & $\boldsymbol{E}_{\mathrm{T}}>280 \mathrm{GeV}$ \\
Cut 6 & $\mathbb{E}_{\mathrm{T}} / H_{\mathrm{T}}^{\text {lep }}>\max \left(5,15-2 m_{\ell \ell} / \mathrm{GeV}\right)$ \\
\hline \hline
\end{tabular}

a larger $M_{X}$ gives smaller mass splittings. This can be seen by comparing BMP3 to BMP1 or BMP4 to BMP2.

For a $p p$ collider at $\sqrt{s}=100 \mathrm{TeV}$, we adopt the following reconstruction conditions with higher $p_{\mathrm{T}}$ thresholds than those used at the LHC.

(i) Reconstructed electrons are required to have $p_{\mathrm{T}}>$ $10 \mathrm{GeV}$ and $|\eta|<2.47$.

(ii) Reconstructed muons are required to have $p_{\mathrm{T}}>$ $10 \mathrm{GeV}$ and $|\eta|<2.5$.

(iii) Reconstructed non- $b$-tagged jets are required to have $p_{\mathrm{T}}>60 \mathrm{GeV}$ and $|\eta|<2.8$.

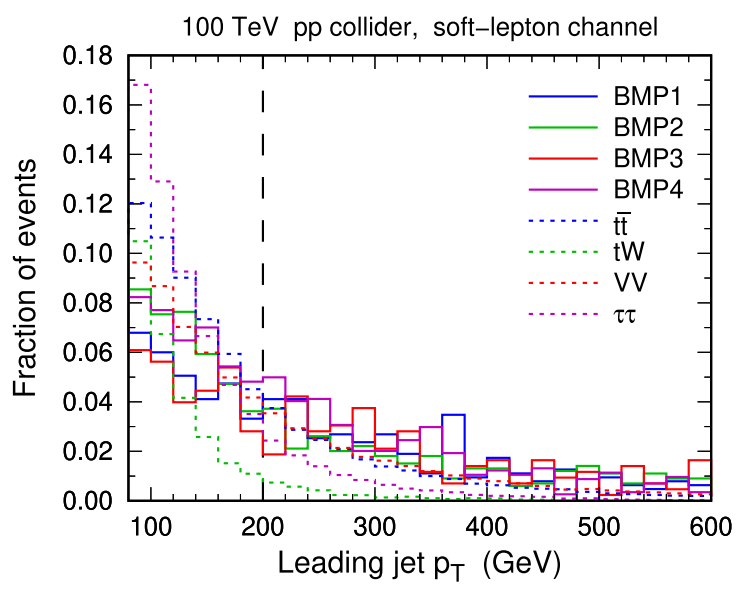

(a) Leading jet $p_{\mathrm{T}}$ distributions (iv) Reconstructed $b$-tagged jets are required to have $p_{\mathrm{T}}>40 \mathrm{GeV}$ and $|\eta|<2.5$.

We appropriately modify the cut conditions according to a collision energy of $100 \mathrm{TeV}$. They are classified into six subsequent cuts, as tabulated in Table VII. The $m_{\mathrm{T}}^{\ell_{1}}$ cut is abandoned, as we find that it would not be helpful.

Cut 1 selects the events with a proper soft SFOS lepton pair. After applying cut 1, the fraction of events binned in the leading jet $p_{\mathrm{T}}$ for the four BMPs and for the backgrounds $t \bar{t}+$ jets, $t W+$ jets, $V V+$ jets, and $\tau^{+} \tau^{-}+$jets are presented in Fig. 7(a). We can see that these backgrounds tend to have lower $p_{\mathrm{T}}$. Thus, we require the leading jet $p_{\mathrm{T}}>200 \mathrm{GeV}$ in cut 2 for reducing the backgrounds.

Figure 7(b) shows the $m_{\tau \tau}$ distributions of signal and background events after cut 2 . The $m_{\tau \tau}$ variable is defined by $m_{\tau \tau}=\operatorname{sgn}\left(m_{\tau \tau}^{2}\right) \sqrt{\left|m_{\tau \tau}^{2}\right|}$ with $m_{\tau \tau}^{2} \equiv\left(1+\xi_{1}\right)\left(1+\xi_{2}\right) m_{\ell \ell}^{2}$, where $\xi_{1}$ and $\xi_{2}$ are parameters determined by solving $\not p_{\mathrm{T}}=\xi_{1} \mathbf{p}_{\mathrm{T}}^{\ell_{1}}+\xi_{2} \mathbf{p}_{\mathrm{T}}^{\ell_{2}}$ event by event $[53,54,74,75]$. If the $\tau$ leptons in the $p p \rightarrow Z^{(*)} / \gamma^{*}\left(\rightarrow \tau^{+} \tau^{-}\right)+$jets process both decay leptonically and the daughter neutrinos are collinear with the daughter charged leptons, such a $m_{\tau \tau}$ definition will truly correspond to the invariant mass of the $\tau$ leptons when the missing transverse momentum $\not_{\mathrm{T}}$ is genuinely contributed by the neutrinos. Such a collinear situation would be realized when the two $\tau$ leptons are sufficiently

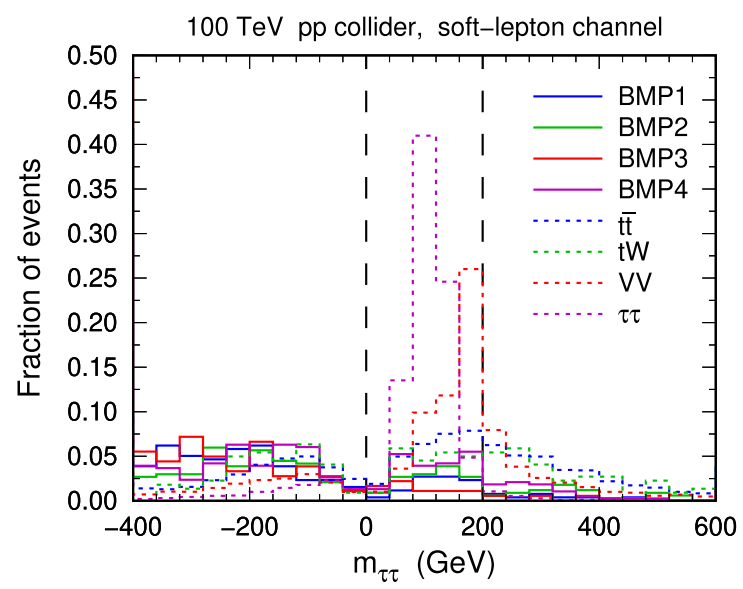

(b) $m_{\tau \tau}$ distributions

FIG. 7. Fraction of signal and background events binned in the leading jet $p_{\mathrm{T}}$ after cut 1 (a) and in $m_{\tau \tau}$ after cut 2 (b) in the soft-leptons + jets $+\mathscr{E}_{\mathrm{T}}$ channel at a $100 \mathrm{TeV}$ p p collider. Dashed lines indicate the cut thresholds. 


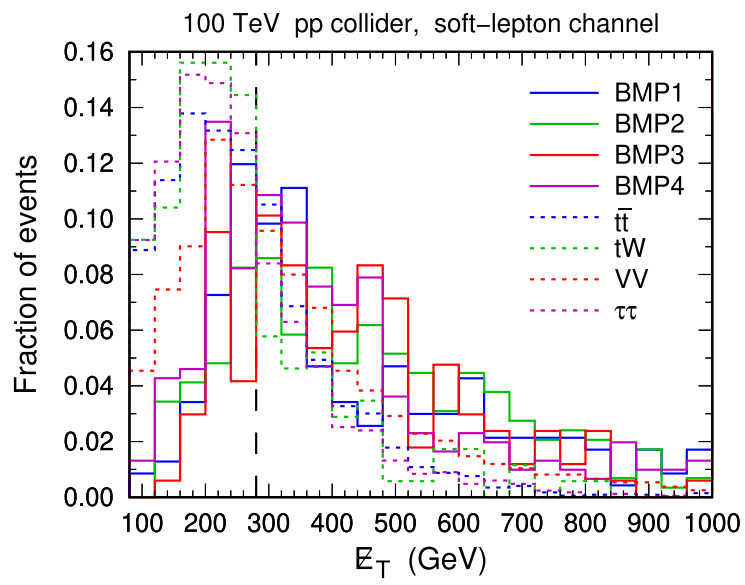

(a) $\mathbb{E}_{\mathrm{T}}$ distributions

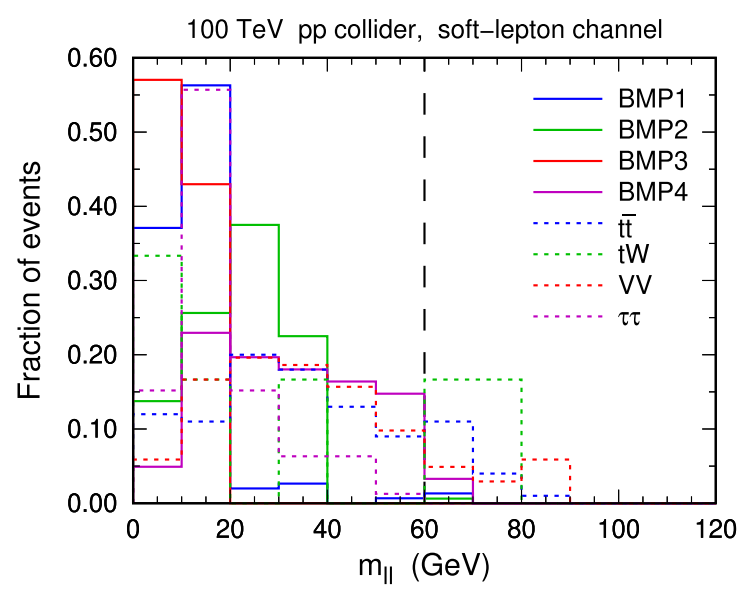

(b) $m_{\ell \ell}$ distributions

FIG. 8. Fraction of signal and background events binned in $\mathbb{E}_{\mathrm{T}}$ after cut 3 (a) and in $m_{\ell \ell}$ after cut 5 (b) in the soft-leptons + jets $+\mathbb{E}_{\mathrm{T}}$ channel at a $100 \mathrm{TeV} p p$ collider. Dashed lines indicate the cut thresholds.

boosted. Consequently, the $m_{\tau \tau}$ distribution of the $\tau^{+} \tau^{-}+$ jets background peaks around $m_{Z}$, as demonstrated in Fig. 7(b). Additionally, the $V V+$ jets distribution peaks around $2 m_{W}$ because of the $W^{+} W^{-} \rightarrow \tau^{+} \tau^{-} \nu_{\tau} \bar{\nu}_{\tau}$ decay process. Therefore, a veto on the events with $m_{\tau \tau} \in$ $[0,200] \mathrm{GeV}$ in cut 3 can significantly suppress the $\tau^{+} \tau^{-}+$ jets and $V V+$ jets backgrounds.

Figure 8(a) presents the $\mathbb{E}_{\mathrm{T}}$ distributions after applying cut 3 . We find that the signal distributions are typically harder than the backgrounds, because the DM candidate $\phi$ with a mass of $\mathcal{O}\left(10^{2}\right) \mathrm{GeV}$ induces larger $\mathbb{E}_{\mathrm{T}}$ than neutrinos. Thus, we adopt the condition $\mathbb{E}_{\mathrm{T}}>280 \mathrm{GeV}$ in cut 4 to increase the signal significance. Cut 5 and cut 6 make use of the $E_{\mathrm{T}} / H_{\mathrm{T}}^{\text {lep }}$ and $m_{\ell \ell}$ variables, following the ATLAS analysis [74].

The $m_{\ell \ell}$ distributions after applying cut 5 are displayed in Fig. 8(b). Inferring from Table VI, we have $m_{a}-m_{\phi} \sim 20,39,16$, and $69 \mathrm{GeV}$ for BMP1, BMP2, BMP3, and BMP4, respectively. Such a difference in the mass splitting results in different end points in the $m_{\ell \ell}$ distributions, as clearly shown in Fig. 8(b). Seven signal regions are defined by the $m_{\ell \ell}$ bins as the same as those in
Table V. Different $m_{\ell \ell}$ bins would be suitable for different mass splittings.

Table VIII lists the visible cross section and the signal significance for an integrated luminosity of $3 \mathrm{ab}^{-1}$ after applying each cut in SR3. We can see that the signal significances of the four BMPs subsequently increase from cut 1 to cut 5 . The cut condition $m_{\ell \ell} \in[1,3] \cup$ $[3.2,10] \mathrm{GeV}$ in SR3 increases the significances of BMP1 and BMP 3 but decreases those of BMP2 and BMP4. This is because BMP1 and BMP3 have smaller mass splittings and, hence, sufficient fractions of events satisfying $m_{\ell \ell} \leq 10 \mathrm{GeV}$, while BMP2 and BMP4 do not, as shown in Fig. 8(b). Larger $m_{\ell \ell}$ bins in SR6 and SR7 would be applicable for BMP2 and BMP4.

Figure 9 shows the 95\% C.L. expected exclusion region combing the seven signal regions at a $100 \mathrm{TeV} p p$ collider with a dataset of $3 \mathrm{ab}^{-1}$ for the fixed coupling relation $\lambda_{1}=\lambda_{2}=3 \lambda_{0} / 2$. We find that the soft-leptons + jets $+\mathscr{E}_{\mathrm{T}}$ channel can explore a region up to $m_{\phi} \sim 550 \mathrm{GeV}$. Nonetheless, such a sensitivity is not better than that in the monojet $+\mathscr{E}_{\mathrm{T}}$ channel, which is demonstrated by the red dashed line.

TABLE VIII. Visible cross section $\sigma_{\text {vis }}$ in femtobarns and signal significance $\mathcal{S}$ for integrated luminosity $3 \mathrm{ab}^{-1}$ after each cut in SR3 of the soft-leptons + jets $+\mathbb{E}_{\mathrm{T}}$ channel at $\sqrt{s}=100 \mathrm{TeV}$.

\begin{tabular}{|c|c|c|c|c|c|c|c|c|c|c|c|c|}
\hline & \multirow{2}{*}{$\frac{t \bar{t}}{\sigma_{\mathrm{vis}}}$} & \multirow{2}{*}{$\begin{array}{l}t W \\
\sigma_{\text {vis }}\end{array}$} & \multirow{2}{*}{$\frac{V V}{\sigma_{\text {vis }}}$} & \multirow{2}{*}{$\begin{array}{c}\tau \tau \\
\sigma_{\mathrm{vis}}\end{array}$} & \multicolumn{2}{|c|}{ BMP1 } & \multicolumn{2}{|c|}{ BMP2 } & \multicolumn{2}{|c|}{ BMP3 } & \multicolumn{2}{|c|}{ BMP4 } \\
\hline & & & & & $\sigma_{\mathrm{vis}}$ & $\mathcal{S}$ & $\sigma_{\text {vis }}$ & $\mathcal{S}$ & $\sigma_{\text {vis }}$ & $\mathcal{S}$ & $\sigma_{\text {vis }}$ & $\mathcal{S}$ \\
\hline Cut 1 & 37600 & 28400 & 5070 & 5420 & 1.53 & 0.303 & 2.29 & 0.453 & 0.618 & 0.122 & 17.3 & 3.43 \\
\hline Cut 2 & 1790 & 296 & 804 & 510 & 0.625 & 0.586 & 0.770 & 0.722 & 0.262 & 0.246 & 5.77 & 5.42 \\
\hline Cut 3 & 1280 & 232 & 383 & 73.3 & 0.567 & 0.699 & 0.669 & 0.825 & 0.243 & 0.300 & 4.61 & 5.68 \\
\hline Cut 4 & 445 & 69.7 & 190 & 21.7 & 0.426 & 0.863 & 0.531 & 1.08 & 0.201 & 0.408 & 3.14 & 6.35 \\
\hline Cut 5 & 37.3 & 8.04 & 9.91 & 3.47 & 0.366 & 2.57 & 0.368 & 2.59 & 0.185 & 1.30 & 0.934 & 6.50 \\
\hline SR3 & 4.11 & 2.68 & 0.583 & 0.528 & 0.136 & 2.59 & 0.0483 & 0.921 & 0.106 & 2.02 & 0.0455 & 0.868 \\
\hline
\end{tabular}




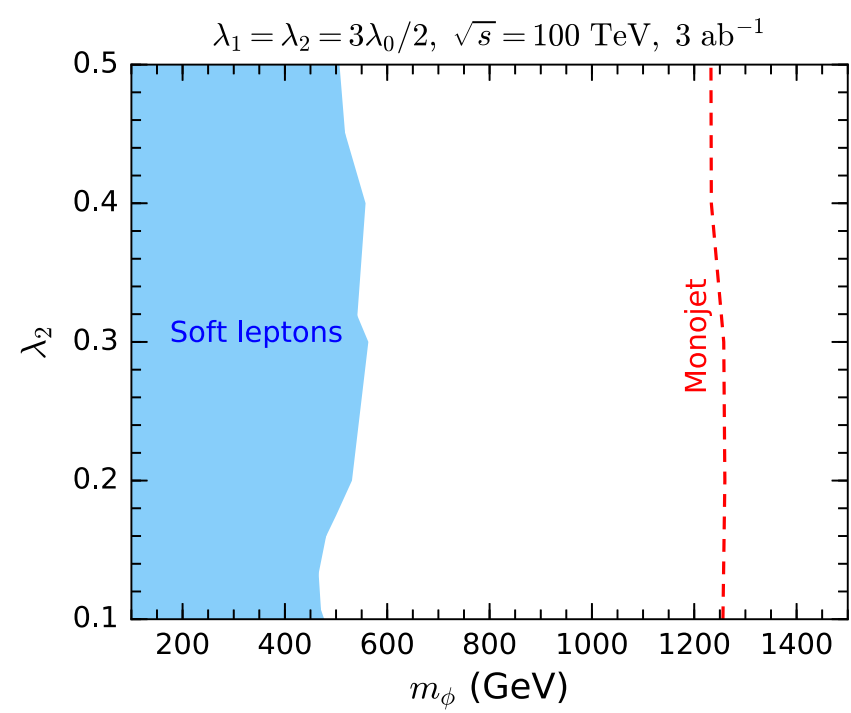

FIG. 9. 95\% C.L. expected exclusion region in the soft-leptons + jets $+\mathscr{E}_{\mathrm{T}}$ channel at a $100 \mathrm{TeV} p$ p collider with an integrated luminosity of $3 \mathrm{ab}^{-1}$ for the fixed coupling relation $\lambda_{1}=\lambda_{2}=3 \lambda_{0} / 2$. For comparison, the red dashed line denotes the 95\% C.L. expected exclusion limit in the monojet $+\mathscr{E}_{\mathrm{T}}$ channel with the same collision energy and integrated luminosity.

\section{CONCLUSIONS AND DISCUSSIONS}

In this paper, we discuss the QSDM model, where the dark sector contains an inert $\mathrm{SU}(2)_{\mathrm{L}}$ quadruplet scalar with $Y=1 / 2$. After the electroweak symmetry breaking, there are one doubly charged scalar, two singly charged scalars, and two neutral scalars. For $\lambda_{2}>0$, the lighter neutral scalar $\phi$ plays the role of DM particle. We have identified the parameter regions that can predict an observed DM relic abundance.

As the DM candidate can interact with nucleons through the SM Higgs portal, direct detection experiments could be sensitive to this model. We have investigated the constraints from the current experiment XENON1T as well as the sensitivity of the future LZ experiment. Nonetheless, the $h \phi \phi$ coupling could vanish if the quartic couplings $\lambda_{0}, \lambda_{1}$, and $\lambda_{2}$ satisfy special relations, resulting in null signal in direct detection. In this case, other types of DM search experiments would be essentially important.

Since the dark sector scalars carry electroweak charges, they could be directly produced in pairs at high-energy $p p$ colliders. The mass splittings among the dark sector scalars are typically lower than $m_{W}$ and $m_{Z}$. As a result, the sensitive search channels at the LHC include the monojet + $\mathscr{E}_{\mathrm{T}}$ and soft-leptons + jets $+\mathbb{E}_{\mathrm{T}}$ channels. We have recast the ATLAS analyses in these two channels with $\sqrt{s}=$ $13 \mathrm{TeV}$ and an integrated luminosity of $36.1 \mathrm{fb}^{-1}$. We have found that the monojet search has excluded some parameter regions up to $m_{\phi} \sim 45 \mathrm{GeV}$, while the soft-lepton channel has excluded larger regions up to $m_{\phi} \sim 85 \mathrm{GeV}$.

As these LHC constraints on the QSDM model still seem rather weak, we have studied the prospect of a future $100 \mathrm{TeV} p p$ collider, either SPPC or FCC-hh. We have found that the monojet channel could be sensitive to the model up to $m_{\phi} \sim 1.2 \mathrm{TeV}$ assuming an integrated luminosity of $3 \mathrm{ab}^{-1}$. On the other hand, the soft-lepton channel is less sensitive, reaching up to $m_{\phi} \sim 550 \mathrm{GeV}$.

Electroweak precision measurements provide an indirect probe to the QSDM model. The future determination of electroweak oblique parameters in the CEPC project would be able to reach up to $m_{\phi} \sim 0.6-1.3 \mathrm{TeV}$. But a direct search in the monojet channel at a $100 \mathrm{TeV} p p$ collider seems more sensitive in most regions.

Compared to the IDM, the QSDM model involves more electroweakly interacting dark sector scalars living in a larger $\mathrm{SU}(2)_{\mathrm{L}}$ representation. This effectively enhances the annihilation and coannihilation cross sections of the scalars in the early Universe. As a result, higher mass scales $(\gtrsim 2-3 \mathrm{TeV})$ are required to yield the observed DM relic abundance. Another consequence is that the pair production rates of the scalars at $p p$ colliders significantly increase. Therefore, the LHC and a $100 \mathrm{TeV} p p$ collider are able to probe higher mass scales in the QSDM model than in the IDM (cf. Refs. [59,76-78]).

\section{ACKNOWLEDGMENTS}

This work is supported in part by the National Natural Science Foundation of China under Grants No. 11805288, No. 11875327, and No. 11905300, the China Postdoctoral Science Foundation under Grant No. 2018M643282, the Natural Science Foundation of Guangdong Province under Grant No. 2016A030313313, the Fundamental Research Funds for the Central Universities, and the Sun Yat-Sen University Science Foundation.

\section{APPENDIX: ELECTROWEAK GAUGE INTERACTIONS OF THE QUADRUPLET SCALAR}

The generators in the $\mathrm{SU}(2)_{\mathrm{L}}$ representation 4 are given by

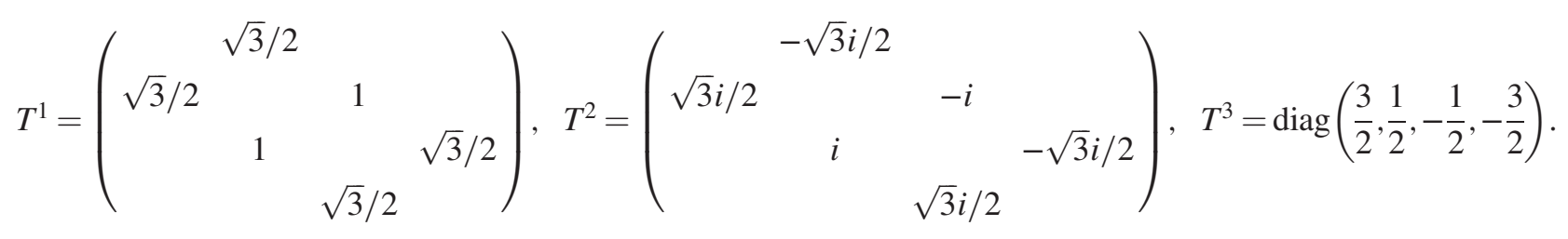


Utilizing these generators, we can expand the gauge interaction terms for the quadruplet scalar as

$$
\begin{aligned}
& \mathcal{L}_{\text {gauge }}=g\left[\frac{\sqrt{6}}{2} W_{\mu}^{+}\left(X^{++}\right)^{*} i \stackrel{\leftrightarrow}{\partial^{\mu}} X^{+}+\sqrt{2} W_{\mu}^{+}\left(X^{+}\right)^{*} i \stackrel{\leftrightarrow}{\partial^{\mu}} X^{0}+\frac{\sqrt{6}}{2} W_{\mu}^{+}\left(X^{0}\right)^{*} i \stackrel{\leftrightarrow}{\partial^{\mu}} X^{-}+\text {H.c. }\right] \\
& +e A_{\mu}\left[2\left(X^{++}\right)^{*} i \stackrel{\leftrightarrow}{\partial^{\mu}} X^{++}+\left(X^{+}\right)^{*} i \stackrel{\leftrightarrow}{\partial^{\mu}} X^{+}-\left(X^{-}\right)^{*} i \stackrel{\leftrightarrow}{\partial^{\mu}} X^{-}\right] \\
& +\frac{g}{2 c_{\mathrm{W}}} Z_{\mu}\left[\left(3 c_{\mathrm{W}}^{2}-s_{\mathrm{W}}^{2}\right)\left(X^{++}\right)^{*} i \stackrel{\leftrightarrow}{\partial^{\mu}} X^{++}+\left(c_{\mathrm{W}}^{2}-s_{\mathrm{W}}^{2}\right)\left(X^{+}\right)^{*} i \stackrel{\leftrightarrow}{\partial^{\mu}} X^{+}\right. \\
& \left.+i a i \stackrel{\leftrightarrow}{\partial^{\mu}} \phi-\left(3 c_{\mathrm{W}}^{2}+s_{\mathrm{W}}^{2}\right)\left(X^{-}\right)^{*} i \stackrel{\leftrightarrow}{\partial^{\mu}} X^{-}\right] \\
& +\frac{g^{2}}{2} W_{\mu}^{+} W^{-\mu}\left[3\left|X^{++}\right|^{2}+7\left|X^{+}\right|^{2}+7\left|X^{0}\right|^{2}+3\left|X^{-}\right|^{2}\right] \\
& +g^{2}\left[\sqrt{3} W_{\mu}^{+} W^{+\mu}\left(X^{++}\right)^{*} X^{0}+\sqrt{3} W_{\mu}^{+} W^{+\mu}\left(X^{+}\right)^{*} X^{-}+\text {H.c. }\right] \\
& +e^{2} A_{\mu} A^{\mu}\left(4\left|X^{++}\right|^{2}+\left|X^{+}\right|^{2}+\left|X^{-}\right|^{2}\right) \\
& +\frac{e g}{c_{\mathrm{W}}} A_{\mu} Z^{\mu}\left[2\left(3 c_{\mathrm{W}}^{2}-s_{\mathrm{W}}^{2}\right)\left|X^{++}\right|^{2}+\left(c_{\mathrm{W}}^{2}-s_{\mathrm{W}}^{2}\right)\left|X^{+}\right|^{2}+\left(3 c_{\mathrm{W}}^{2}+s_{\mathrm{W}}^{2}\right)\left|X^{-}\right|^{2}\right] \\
& +\frac{g^{2}}{4 c_{\mathrm{W}}^{2}} Z_{\mu} Z^{\mu}\left[\left(3 c_{\mathrm{W}}^{2}-s_{\mathrm{W}}^{2}\right)^{2}\left|X^{++}\right|^{2}+\left(c_{\mathrm{W}}^{2}-s_{\mathrm{W}}^{2}\right)^{2}\left|X^{+}\right|^{2}+\left|X^{0}\right|^{2}+\left(3 c_{\mathrm{W}}^{2}+s_{\mathrm{W}}^{2}\right)^{2}\left|X^{-}\right|^{2}\right] \\
& +\left\{\left[\frac{3 \sqrt{6}}{2} e g A^{\mu}+\frac{\sqrt{6} g^{2}\left(2 c_{\mathrm{W}}^{2}-s_{\mathrm{W}}^{2}\right)}{2 c_{\mathrm{W}}} Z^{\mu}\right] W_{\mu}^{+}\left(X^{++}\right)^{*} X^{+}\right. \\
& +\left(\sqrt{2} e g A^{\mu}-\frac{\sqrt{2} g^{2} s_{\mathrm{W}}^{2}}{c_{\mathrm{W}}} Z^{\mu}\right) W_{\mu}^{+}\left(X^{+}\right)^{*} X^{0} \\
& \left.-\left[\frac{\sqrt{6}}{2} e g A^{\mu}+\frac{\sqrt{6} g^{2}\left(2 c_{\mathrm{W}}^{2}+s_{\mathrm{W}}^{2}\right)}{2 c_{\mathrm{W}}} Z^{\mu}\right] W_{\mu}^{+}\left(X^{0}\right)^{*} X^{-}+\text {H.c. }\right\} \text {. }
\end{aligned}
$$

Here $c_{\mathrm{W}} \equiv \cos \theta_{\mathrm{W}}$ and $s_{\mathrm{W}} \equiv \sin \theta_{\mathrm{W}}$, where $\theta_{\mathrm{W}}=\tan ^{-1}\left(g^{\prime} / g\right)$ is the weak mixing angle.

[1] G. Bertone, D. Hooper, and J. Silk, Particle dark matter: Evidence, candidates and constraints, Phys. Rep. 405, 279 (2005).

[2] J. L. Feng, Dark matter candidates from particle physics and methods of detection, Annu. Rev. Astron. Astrophys. 48, 495 (2010).

[3] B.-L. Young, A survey of dark matter and related topics in cosmology, Front. Phys. (Beijing) 12, 121201 (2017); Erratum, Front. Phys. (Beijing) 12, 121202 (2017).

[4] R. Mahbubani and L. Senatore, The Minimal model for dark matter and unification, Phys. Rev. D 73, 043510 (2006).

[5] M. Cirelli, N. Fornengo, and A. Strumia, Minimal dark matter, Nucl. Phys. B753, 178 (2006).

[6] T. Hambye, F. S. Ling, L. Lopez Honorez, and J. Rocher, Scalar multiplet dark matter, J. High Energy Phys. 07 (2009) 090; Erratum, 05 (2010) 066.

[7] T. Cohen, J. Kearney, A. Pierce, and D. Tucker-Smith, Singlet-doublet dark matter, Phys. Rev. D 85, 075003 (2012).
[8] Y. Cai, W. Chao, and S. Yang, Scalar septuplet dark matter and enhanced $h \rightarrow \gamma \gamma$ decay rate, J. High Energy Phys. 12 (2012) 043.

[9] K. Earl, K. Hartling, H.E. Logan, and T. Pilkington, Constraining models with a large scalar multiplet, Phys. Rev. D 88, 015002 (2013).

[10] S. S. AbdusSalam and T. A. Chowdhury, Scalar representations in the light of electroweak phase transition and cold dark matter phenomenology, J. Cosmol. Astropart. Phys. 05 (2014) 026.

[11] O. Fischer and J. J. van der Bij, The scalar singlet-triplet dark matter model, J. Cosmol. Astropart. Phys. 01 (2014) 032.

[12] A. Dedes and D. Karamitros, Doublet-triplet fermionic dark matter, Phys. Rev. D 89, 115002 (2014).

[13] B. Ostdiek, Constraining the minimal dark matter fiveplet with LHC searches, Phys. Rev. D 92, 055008 (2015).

[14] C. Cai, Z.-M. Huang, Z. Kang, Z.-H. Yu, and H.-H. Zhang, Perturbativity limits for scalar minimal dark matter with 
Yukawa interactions: Septuplet, Phys. Rev. D 92, 115004 (2015).

[15] T. M. P. Tait and Z.-H. Yu, Triplet-quadruplet dark matter, J. High Energy Phys. 03 (2016) 204.

[16] S. Banerjee, S. Matsumoto, K. Mukaida, and Y.-L. S. Tsai, WIMP dark matter in a well-tempered regime: A case study on Singlet-Doublets Fermionic WIMP, J. High Energy Phys. 11 (2016) 070.

[17] W.-B. Lu and P.-H. Gu, Mixed inert scalar triplet dark matter, radiative neutrino masses and leptogenesis, Nucl. Phys. B924, 279 (2017).

[18] C. Cai, Z.-H. Yu, and H.-H. Zhang, CEPC precision of electroweak oblique parameters and weakly interacting dark matter: The fermionic case, Nucl. Phys. B921, 181 (2017).

[19] C. Cai, Z.-H. Yu, and H.-H. Zhang, CEPC precision of electroweak oblique parameters and weakly interacting dark matter: The scalar case, Nucl. Phys. B924, 128 (2017).

[20] X. Liu and L. Bian, Dark matter and electroweak phase transition in the mixed scalar dark matter model, Phys. Rev. D 97, 055028 (2018).

[21] Q.-F. Xiang, X.-J. Bi, P.-F. Yin, and Z.-H. Yu, Exploring fermionic dark matter via Higgs boson precision measurements at the circular electron positron collider, Phys. Rev. D 97, 055004 (2018).

[22] C. Cai, Z. Kang, Z. Luo, Z.-H. Yu, and H.-H. Zhang, Scalar quintuplet minimal dark matter with Yukawa interactions: Perturbative up to the Planck scale, Chin. Phys. C 43, 023102 (2019).

[23] L. Lopez Honorez, M. H. G. Tytgat, P. Tziveloglou, and B. Zaldivar, On minimal dark matter coupled to the Higgs, J. High Energy Phys. 04 (2018) 011.

[24] C. Cai, Z. Kang, H.-H. Zhang, and Y.-P. Zeng, Minimal dark matter in $S U(2)_{L} \times U(1)_{Y} \times U(1)_{B-L}$, Phys. Lett. B 784, 385 (2018).

[25] A. Dutta Banik, A. K. Saha, and A. Sil, Scalar assisted singlet doublet fermion dark matter model and electroweak vacuum stability, Phys. Rev. D 98, 075013 (2018).

[26] P.-H. Gu and H.-J. He, TeV scale neutrino mass generation, minimal inelastic dark matter, and high scale leptogenesis, Phys. Rev. D 99, 015025 (2019).

[27] A. Betancur and Ó. Zapata, Phenomenology of doublettriplet fermionic dark matter in nonstandard cosmology and multicomponent dark sectors, Phys. Rev. D 98, 095003 (2018).

[28] K. Kadota and A. Spray, Electroweak multiplet dark matter at future lepton colliders, J. High Energy Phys. 02 (2019) 017.

[29] J.-W. Wang, X.-J. Bi, P.-F. Yin, and Z.-H. Yu, Impact of fermionic electroweak multiplet dark matter on vacuum stability with one-loop matching, Phys. Rev. D 99, 055009 (2019).

[30] A. Filimonova and S. Westhoff, Long live the Higgs portal! J. High Energy Phys. 02 (2019) 140.

[31] W. Chao, G.-J. Ding, X.-G. He, and M. Ramsey-Musolf, Scalar electroweak multiplet dark matter, J. High Energy Phys. 08 (2019) 058.

[32] T. Abe and R. Sato, Current status and future prospects of the singlet-doublet dark matter model with $C P$-violation, Phys. Rev. D 99, 035012 (2019).
[33] Y. Cheng and W. Liao, Fate of false vacuum in a singletdoublet dark matter model with RG improved effective action, Phys. Rev. D 101, 055038 (2020).

[34] N. G. Deshpande and E. Ma, Pattern of symmetry breaking with two Higgs doublets, Phys. Rev. D 18, 2574 (1978).

[35] R. Barbieri, L. J. Hall, and V. S. Rychkov, Improved naturalness with a heavy Higgs: An Alternative road to LHC physics, Phys. Rev. D 74, 015007 (2006).

[36] M. Gustafsson, E. Lundstrom, L. Bergstrom, and J. Edsjo, Significant Gamma Lines from Inert Higgs Dark Matter, Phys. Rev. Lett. 99, 041301 (2007).

[37] Q.-H. Cao, E. Ma, and G. Rajasekaran, Observing the dark scalar doublet and its impact on the standard-model Higgs boson at colliders, Phys. Rev. D 76, 095011 (2007).

[38] P. Fileviez Perez, H. H. Patel, M. Ramsey-Musolf, and K. Wang, Triplet scalars and dark matter at the LHC, Phys. Rev. D 79, 055024 (2009).

[39] T. Araki, C. Q. Geng, and K. I. Nagao, Dark matter in inert triplet models, Phys. Rev. D 83, 075014 (2011).

[40] F.-X. Josse-Michaux and E. Molinaro, Triplet scalar dark matter and leptogenesis in an inverse seesaw model of neutrino mass generation, Phys. Rev. D 87, 036007 (2013).

[41] S. Y. Ayazi and S. M. Firouzabadi, Constraining inert triplet dark matter by the LHC and FermiLAT, J. Cosmol. Astropart. Phys. 11 (2014) 005.

[42] N. Khan, Exploring the hyperchargeless Higgs triplet model up to the Planck scale, Eur. Phys. J. C 78, 341 (2018).

[43] M. Dong et al. (CEPC Study Group Collaboration), CEPC conceptual design report: Volume 2-Physics \& Detector, arXiv: 1811.10545.

[44] M. Beltran, D. Hooper, E. W. Kolb, Z. A. C. Krusberg, and T. M. P. Tait, Maverick dark matter at colliders, J. High Energy Phys. 09 (2010) 037.

[45] A. Rajaraman, W. Shepherd, T. M. P. Tait, and A. M. Wijangco, LHC bounds on interactions of dark matter, Phys. Rev. D 84, 095013 (2011).

[46] P. J. Fox, R. Harnik, J. Kopp, and Y. Tsai, Missing energy signatures of dark matter at the LHC, Phys. Rev. D 85, 056011 (2012).

[47] Z.-H. Yu, X.-J. Bi, Q.-S. Yan, and P.-F. Yin, Detecting light stop pairs in coannihilation scenarios at the LHC, Phys. Rev. D 87, 055007 (2013).

[48] Q.-F. Xiang, X.-J. Bi, P.-F. Yin, and Z.-H. Yu, Searches for dark matter signals in simplified models at future hadron colliders, Phys. Rev. D 91, 095020 (2015).

[49] J.-W. Wang, X.-J. Bi, Q.-F. Xiang, P.-F. Yin, and Z.-H. $\mathrm{Yu}$, Exploring triplet-quadruplet fermionic dark matter at the LHC and future colliders, Phys. Rev. D 97, 035021 (2018).

[50] G. F. Giudice, T. Han, K. Wang, and L.-T. Wang, Nearly degenerate gauginos and dark matter at the LHC, Phys. Rev. D 81, 115011 (2010).

[51] S. Gori, S. Jung, and L.-T. Wang, Cornering electroweakinos at the LHC, J. High Energy Phys. 10 (2013) 191.

[52] P. Schwaller and J. Zurita, Compressed electroweakino spectra at the LHC, J. High Energy Phys. 03 (2014) 060.

[53] Z. Han, G. D. Kribs, A. Martin, and A. Menon, Hunting quasidegenerate Higgsinos, Phys. Rev. D 89, 075007 (2014). 
[54] H. Baer, A. Mustafayev, and X. Tata, Monojet plus soft dilepton signal from light higgsino pair production at LHC14, Phys. Rev. D 90, 115007 (2014).

[55] M. Ahmad et al. (CEPC-SPPC Study Group Collaboration), CEPC-SPPC preliminary conceptual design report. 1. Physics and Detector, Reports No. IHEP-CEPC-DR-2015-01, No. IHEP-TH-2015-01, and No. IHEP-EP-2015-01.

[56] A. Abada et al. (FCC Collaboration), FCC Physics Opportunities, Eur. Phys. J. C 79, 474 (2019).

[57] Y. Hamada, K. Kawana, and K. Tsumura, Landau pole in the Standard Model with weakly interacting scalar fields, Phys. Lett. B 747, 238 (2015).

[58] G. Degrassi, S. Di Vita, J. Elias-Miro, J. R. Espinosa, G. F. Giudice, G. Isidori, and A. Strumia, Higgs mass and vacuum stability in the Standard Model at NNLO, J. High Energy Phys. 08 (2012) 098.

[59] A. Belyaev, G. Cacciapaglia, I. P. Ivanov, F. Rojas-Abatte, and M. Thomas, Anatomy of the inert two Higgs doublet model in the light of the LHC and non-LHC dark matter searches, Phys. Rev. D 97, 035011 (2018).

[60] K. Griest and D. Seckel, Three exceptions in the calculation of relic abundances, Phys. Rev. D 43, 3191 (1991).

[61] A. Alloul, N. D. Christensen, C. Degrande, C. Duhr, and B. Fuks, FeynRules 2.0-A complete toolbox for tree-level phenomenology, Comput. Phys. Commun. 185, 2250 (2014).

[62] J. Alwall, R. Frederix, S. Frixione, V. Hirschi, F. Maltoni, O. Mattelaer, H. S. Shao, T. Stelzer, P. Torrielli, and M. Zaro, The automated computation of tree-level and next-toleading order differential cross sections, and their matching to parton shower simulations, J. High Energy Phys. 07 (2014) 079.

[63] F. Ambrogi, C. Arina, M. Backovic, J. Heisig, F. Maltoni, L. Mantani, O. Mattelaer, and G. Mohlabeng, MadDM v.3.0: A comprehensive tool for dark matter studies, Phys. Dark Universe 24, 100249 (2019).

[64] N. Aghanim et al. (Planck Collaboration), Planck 2018 results. VI. Cosmological parameters, arXiv:1807.06209.

[65] E. Aprile et al. (XENON Collaboration), Dark Matter Search Results from a One Ton-Year Exposure of XENON1T, Phys. Rev. Lett. 121, 111302 (2018).

[66] B. J. Mount et al., LUX-ZEPLIN (LZ) Technical Design Report, arXiv:1703.09144.

[67] Z.-H. Yu, J.-M. Zheng, X.-J. Bi, Z. Li, D.-X. Yao, and H.-H. Zhang, Constraining the interaction strength between dark matter and visible matter: II. scalar, vector and spin-3/2 dark matter, Nucl. Phys. B860, 115 (2012).

[68] J. R. Ellis, A. Ferstl, and K. A. Olive, Reevaluation of the elastic scattering of supersymmetric dark matter, Phys. Lett. B 481, 304 (2000).

[69] M. Aaboud et al. (ATLAS Collaboration), Search for dark matter and other new phenomena in events with an energetic jet and large missing transverse momentum using the ATLAS detector, J. High Energy Phys. 01 (2018) 126.

[70] T. Sjöstrand, S. Ask, J. R. Christiansen, R. Corke, N. Desai, P. Ilten, S. Mrenna, S. Prestel, C. O. Rasmussen, and P. Z. Skands, An Introduction to PYTHIA8.2, Comput. Phys. Commun. 191, 159 (2015).

[71] M. L. Mangano, M. Moretti, F. Piccinini, and M. Treccani, Matching matrix elements and shower evolution for topquark production in hadronic collisions, J. High Energy Phys. 01 (2007) 013.

[72] J. de Favereau, C. Delaere, P. Demin, A. Giammanco, V. Lemaître, A. Mertens, and M. Selvaggi (DELPHES3 Collaboration), DELPHES3, A modular framework for fast simulation of a generic collider experiment, J. High Energy Phys. 02 (2014) 057.

[73] M. Aaboud et al. (ATLAS Collaboration), Search for electroweak production of supersymmetric particles in final states with two or three leptons at $\sqrt{s}=13 \mathrm{TeV}$ with the ATLAS detector, Eur. Phys. J. C 78, 995 (2018).

[74] M. Aaboud et al. (ATLAS Collaboration), Search for electroweak production of supersymmetric states in scenarios with compressed mass spectra at $\sqrt{s}=13 \mathrm{TeV}$ with the ATLAS detector, Phys. Rev. D 97, 052010 (2018).

[75] A. Barr and J. Scoville, A boost for the EW SUSY hunt: Monojet-like search for compressed sleptons at LHC14 with $100 \mathrm{fb}^{-1}$, J. High Energy Phys. 04 (2015) 147.

[76] A. Arhrib, Y.-L. S. Tsai, Q. Yuan, and T.-C. Yuan, An updated analysis of inert Higgs doublet model in light of the recent results from LUX, PLANCK, AMS-02 and LHC, J. Cosmol. Astropart. Phys. 06 (2014) 030.

[77] A. Datta, N. Ganguly, N. Khan, and S. Rakshit, Exploring collider signatures of the inert Higgs doublet model, Phys. Rev. D 95, 015017 (2017).

[78] A. Belyaev, T. F. P. Tomei, P. Mercadante, C. Moon, S. Moretti, S. Novaes, L. Panizzi, F. Rojas, and M. Thomas, Advancing LHC probes of dark matter from the inert twoHiggs-doublet model with the monojet signal, Phys. Rev. D 99, 015011 (2019). 